\title{
The Double-Wedge Algebra for Quantum Fields on Schwarzschild and Minkowski Spacetimes *
}

\author{
Bernard S. Kay \\ Institut für Theoretische Physik, Universität Zürich, Schönberggasse 9, CH-8001 Zürich, \\ Switzerland
}

\begin{abstract}
We consider the Klein-Gordon equation $(m \geqq 0)$ on the double Schwarzschild wedge of the Kruskal spacetime, and construct the HartleHawking state $\omega_{H}$ as a thermal state relative to the Boulware quantization. We prove that, on the double wedge, $\omega_{H}$ is a pure state, and in the corresponding representation, the left- and right-wedge $C^{*}$ algebras each have the ReehSchlieder property, while the corresponding von-Neumann algebras are type $I I I_{1}$ factors which are dual to (i.e. commutants of) each other. We discuss the extent to which these properties may generalize to non-quasi-free field theories.

Pursuing the Rindler-Fulling-Unruh analogy with the Klein-Gordon equation $(m>0)$ in ( $d$-dimensional) flat spacetime, we establish an explicit formula for the Minkowski vacuum on a spacelike double wedge as a thermal state relative to the Fulling quantization. We also treat the case $d=2, m=0$ of this formula since this is essential input for a paper with Dimock on scattering theory for the quantum Klein-Gordon equation on the Schwarzschild metric.
\end{abstract}

\section{Contents}

0. Introduction . . . . . . . . . . . . . . . . . . . . . . 58

1. Preliminaries . . . . . . . . . . . . . . . . . . . . . . . . . . . . . . . 59

2. The Minkowski Vacuum . . . . . . . . . . . . . . . . . . . 63

3. The Hartle-Hawking State . . . . . . . . . . . . . . . . . . . . . . . . . 67

4. Construction of (Regular) Fulling and Boulware Ground One-Particle Structures . . 70

5. Discussion . . . . . . . . . . . . . . . . . . . . . . . . . . . 73

A1. One-Particle Equivalents to the Reeh-Schlieder and Bisognano-Wichmann Theorems. . . . . . . . . . . . . . . . . . . . . . . . . . . . . . . . . 74

A2. Notes on the Minkowski Cases $m=0, d \neq 4$ (Especially $m=0$ and $d=2$ ) . . . . . . 76

A3. A proof that $\mathrm{sp}\left(h_{\mathrm{B}}\right)=[0, \infty)$. . . . . . . . . . . . . . . . . . . . . . 77

A4. Some Results on Self-Adjoint and Positive-Self-Adjoint Operators Required for Sect. 4 . . . . . . . . . . . . . . . . . . . . . . . . . . . . . 79

* Research supported in part by the Schweizerischer Nationalfonds 


\section{Introduction}

After Hawking's prediction of black hole evaporation in 1974 [1], a big effort was devoted to further clarifying the nature of the effect through a systematic study of quantum field theory on background black hole metrics. A picture was built up of the various natural quantum states admitted by ideal black hole metrics and of the relationships between them, and an important analogy emerged (thanks to the work of Fulling [2, 3], Davies [4], Hartle and Hawking [5], Hawking [6], Unruh [7], Israel [8], and others) between quantum field theory on (say) the Schwarzschild metric and quantum field theory in flat spacetime according to the now familiar schema:

$$
\begin{array}{ll}
\text { Kruskal spacetime } & \leftrightarrow \text { Minkowski space } \\
\text { exterior Schwarzschild spacetime } & \leftrightarrow \text { Rindler wedge } \\
\text { Hartle-Hawking state } & \leftrightarrow \text { Minkowski vacuum } \\
\text { Boulware state } & \leftrightarrow \text { Fulling vacuum. }
\end{array}
$$

We recall the basic facts about this schema:

(1) The Boulware state [9] is a ground state for the Schwarzschild time evolution on the exterior Schwarzschild spacetime and is analogous to the Fulling state which is a ground state for wedge-preserving Lorentz boosts on the Rindler wedge of Minkowski space. It is not thought to be a physically realizable state.

(2) The Hartle-Hawking state (which is believed to be the fundamental equilibrium state on the Kruskal spacetime) appears - on the exterior Schwarzschild spacetime of mass ${ }^{1} M$ and relative to the Schwarzschild time evolution - to be a state of thermal equilibrium at the Hawking temperature $T_{H}=(8 \pi M)^{-1}$. Correspondingly, the usual Minkowski vacuum on flat spacetime adopts - when restricted to the Rindler wedge, and relative to wedge-preserving Lorentz boosts the mathematical form of a thermal equilibrium state [with "temperature" $\left.T=(2 \pi)^{-1}\right]$.

Derivations of these properties - for linear fields, and at a heuristic level - may be found in the above quoted literature. (See especially [7]. A comprehensive list of references may be found in the recent monograph [10].) More recently, Sewell [11] has pointed out that the thermal property of the Minkowski vacuum is an immediate consequence of the Bisognano-Wichmann theorem [12]. Sewell went on to exploit the analogy (*) in motivating a set of axioms which are claimed to characterize the Hartle-Hawking state for a general quantum field theory.

The main purpose of the present paper is to explicitly construct the HartleHawking state in the case of a model linear field theory (the covariant KleinGordon equation) and to establish some of its properties.

We begin at the other side of the analogy (*) by constructing, in Sect. 2, the Fulling quantization on the $C^{*}$ algebra of the free Klein-Gordon field $(m>0)$ on a spacelike double wedge of Minkowski space. Assuming the existence of a "Fulling regular ground one-particle structure" to be established in Sect. 4 we construct both a ground state $\omega_{F}$ and a related one-parameter family of states $\tilde{\omega}_{F}^{\beta}$ which are KMS (for inverse temperature $\beta$ ) with respect to the one-parameter family of

1 We use units with $\hbar=c=G=k=1$ 
wedge-preserving Lorentz boosts. (One may regard $\tilde{\omega}_{F}^{\beta}$ as "heated up" Fulling states.) Our main result here is to strengthen the Bisognano-Wichmann-Sewell result in the case of our quasi-free system by showing (with the help of the "preReeh-Schlieder" and "pre-Bisognano-Wichmann" theorems of Sect. A1) that the usual Minkowski vacuum $\omega_{0}$, when restricted to the double-wedge algebra, coincides with $\tilde{\omega}_{F}^{2 \pi}$. [In Sect. A2, we point out that this theorem extends for $m>0$ to $d$-dimensional Minkowski space $(d \geqq 2)$ and also establish a modified version of the theorem for the case $m=0, d=2$.]

We then turn back, in Sect. 3, to the Kruskal spacetime. Following by now wellknown methods [13-15] we construct a $C^{*}$ algebra for the covariant KleinGordon equation on this spacetime. Then, in exact analogy to the construction of $\omega_{F}$ and $\tilde{\omega}_{F}^{\beta}$ on a double wedge in Minkowski space, and again relying on Sect. 4 for the existence of a "Boulware regular ground one-particle structure", we construct the Boulware state $\omega_{B}$, and a family $\tilde{\omega}_{B}^{\beta}$ of heated up Boulware states on the algebra of the double (exterior-Schwarzschild) wedge of Kruskal. Still following the schema $(*)$, we then define the Hartle-Hawking state $\omega_{H}$ on this region to be $\tilde{\omega}_{B}^{8 \pi M}$. With this definition, we are able to show that $\omega_{H}$ shares several mathematical properties with the Minkowski vacuum $\omega_{0}$ on the flat double wedge. Thus we show that, on the full double wedge, $\omega_{H}$ is a pure state, and in the corresponding (GNS) representation, the left- and right-wedge $C^{*}$ algebras each have the ReehSchlieder property while the corresponding von Neumann algebras are type $I I I_{1}$ factors which are dual to (i.e. commutants of) each other. (For the type $I I I_{1}$ property, we use in Sect. A3 a result from the classical scattering theory developed for the covariant Klein-Gordon equation on the Schwarzschild metric by Dimock and the author [16].)

The proof of our results, which is completed in Sects. 4 and A4, draws heavily on two preparatory papers - one on quasi-free KMS states [17], the other on the purification of (general) KMS states [18] - which will be published simultaneously with this paper. We review the essential results from these papers in Parts 1.1-1.4 of the preliminary Sect. 1 .

The present paper is also closely interrelated with the scattering-theory work by Dimock and the author already mentioned. Our result (in the case $d=2, m=0$ treated in Sect. A2), that $\omega_{0}=\tilde{\omega}_{F}^{2 \pi}$ will be essential input for [16], while [16] contains in turn results on the behaviour of $\omega_{H}$ on the horizon and at infinity as well as a discussion of the Unruh state.

Further discussion of the significance of our results and the relation with other work will be given in Sect. 5 .

\section{Preliminaries}

In Sects. 1.1-1.4, we briefly review the essential results that we require concerning quasi-free Bose systems and what we call double KMS states (quasi-free and otherwise). Not all of these results may be found in the existing literature (even allowing for changes in terminology etc.) and we refer to two companion papers $[17,18]$ for further details, references, and proofs. We shall refer here to [17] as I and to $[18]$ as II. 
1.1 .

We say that a quantum dynamical system $(\mathfrak{H}, \alpha(t))\left[\mathfrak{U}\right.$ a $C^{*}$ algebra, $\alpha(t)$ an automorphism group] is (Bose) quasi-free if $\mathfrak{U}$ is generated by objects $W(\Phi), \Phi$ belonging to some symplectic space $(D, \sigma)$, satisfying

$$
W\left(\Phi_{1}\right) W\left(\Phi_{2}\right)=\exp \left(-i \sigma\left(\Phi_{1}, \Phi_{2}\right) / 2\right) W\left(\Phi_{1}+\Phi_{2}\right),
$$

(more precisely, $\mathfrak{U}$ is the Weyl algebra over $(D, \sigma)$ say in the sense of [19]) while $\alpha(t)$ arises from the action $\alpha(t) W(\Phi)=W(\mathscr{T}(t) \Phi)$, where $\mathscr{T}(t)$ is a symplectic group on $(D, \sigma)$. In the case where $(D, \sigma)$ takes the form $(h, 2 \operatorname{Im}\langle\cdot \mid \cdot\rangle)$ for some complex Hilbert space $h$ and $\mathscr{T}(t)$ is a strongly continuous unitary group $e^{-i h t}$ on $h$ with strictly positive ${ }^{2}$ energy $h$, then one may obtain a ground state $\omega_{0}$ on $\mathfrak{A}$ by taking the usual Fock representation $\varrho^{\mathscr{F}}(W(\chi))=W^{\mathscr{F}}(\chi)$ of $\mathscr{A}$ on Fock space $\mathscr{F}(h)$ over $h$ and defining

$$
\omega_{0}(W(\chi))=\left\langle\Omega^{\mathscr{F}} \mid W^{\mathscr{F}}(\chi) \Omega^{\mathscr{F}}\right\rangle_{\mathscr{F}(h)} \quad\left(=\exp \left(-\frac{1}{2}\|\chi\|_{h}^{2}\right),\right.
$$

where $\Omega^{\mathscr{F}}$ is the usual Fock vacuum vector in $\mathscr{F}(h)$. In this representation of $\mathfrak{A}, \alpha(t)$ is implemented by $\Gamma\left(e^{-i h t}\right)$, where $\Gamma$ is the second quantization map which for any unitary (antiunitary) $U$ on $h$ yields a unitary (antiunitary) $\Gamma(U)$ on $\mathscr{F}(h)$ satisfying $\Gamma(U) \Omega^{\mathscr{F}}=\Omega^{\mathscr{F}}$ and $W(U \chi)=\Gamma(U) W(\chi) \Gamma(U)^{-1}$. To extend this construction for a ground state $\omega_{0}$ to more general such quasi-free systems $(\mathfrak{H}, \alpha(t))$ one first seeks a ground one-particle structure over the classical linear dynamical system $(D, \sigma, \mathscr{T}(t))$.

Definition. This consists of a complex Hilbert space $h$; a real-linear map $K$ from $D$ to $h$ with dense range satisfying (symplecticness) $\sigma\left(\Phi_{1}, \Phi_{2}\right)=2 \operatorname{Im}\left\langle K \Phi_{1} \mid K \Phi_{2}\right\rangle$ $\forall \Phi_{1}, \Phi_{2} \in D$; and a strongly continuous unitary group $e^{-i h t}$ on $h$ with strictly positive energy $h$ such that $K \mathscr{T}(t)=e^{-i h t} K$. One then defines $\omega_{0}$ by $\omega_{0}(W(\Phi))$ $=\exp \left(-\frac{1}{2}\|K \Phi\|_{h}^{2}\right)$.

Theorem 1.1. For a given $(D, \sigma, \mathscr{T}(t)),\left(K, h, e^{-i h t}\right)$ is determined uniquely up to equivalence in the sense that any other candidate $\left(K^{\prime}, h^{\prime}, e^{-i h^{\prime} t}\right)$ necessarily has $K^{\prime}=U K, e^{-i h^{\prime} t} U=U e^{-i h t}$ for some unitary (i.e. isomorphism) $U: h \rightarrow h^{\prime}$ ([20], see also I).

In Sect. A3, we shall use this theorem in conjunction with the following (related) result (Theorem 1.2 of [21]):

Theorem 1.2. Let $A$ be a real linear operator on a complex Hilbert space $\mathscr{H}$, and suppose $\left[A, e^{-i B t}\right]=0 \forall t \in \mathbb{R}$ for some strictly positive complex linear operator $B$. Then $A$ is also complex linear.

Finally, we shall call a ground one-particle structure (as above) regular if it satisfies the condition $K D \subset \mathscr{D}\left(h^{-1 / 2}\right)$. The significance of this condition will be explained in Sect. 1.4.

1.2.

In addition to ground states on quantum dynamical systems $(\mathfrak{A}, \alpha(t))$, we shall be particularly interested in double KMS states $\tilde{\omega}^{\beta}$ on double quantum dynamical systems $(\tilde{\mathfrak{U}}, \tilde{\alpha}(t), \imath)$.

2 I.e. $h$ is a positive self-adjoint operator with no zero eigenvalues 
Definition. A double quantum dynamical system $(\tilde{\mathfrak{A}}, \tilde{\alpha}(t), l)$ consists of a $C^{*}$ algebra (with identity 1) $\mathfrak{A}$ which in turn consists of the tensor product $\mathfrak{U}^{L} \otimes \mathfrak{A}^{R}$ of two preferred commuting subalgebras; an automorphism group $\tilde{\alpha}(t)$ of $\mathfrak{Q}$ such that $\tilde{\alpha}(t): \mathfrak{U}^{L} \rightarrow \mathfrak{U}^{L}, \mathfrak{U}^{R} \rightarrow \mathfrak{U}^{R}$; and an involutary ${ }^{3}$ antiautomorphism ${ }^{4} \iota$ of $\tilde{\mathfrak{U}}$ which commutes with $\tilde{\alpha}(t)$ and which maps $\mathfrak{A}^{L} \rightarrow \mathfrak{Q}^{R}, \mathfrak{Q}^{R} \rightarrow \mathfrak{Q}^{L}$.

Definition. A double KMS state $\tilde{\omega}_{\beta}(0<\beta<\infty)$ over a double quantum dynamical $\operatorname{system}(\tilde{\mathcal{A}}, \tilde{\alpha}(t), l)$ is an $\tilde{\alpha}(t)$ - and $l$-invariant state whose GNS triple $(\tilde{\varrho}, \tilde{\mathscr{H}}, \widetilde{\Omega})$ (has $\widetilde{\mathscr{H}}$ separable and) satisfies

(i) $\tilde{\Omega}$ is cyclic for $\tilde{\varrho}\left(\mathfrak{Q}^{R}\right)$ alone, and

(ii) the unique unitary implementor of $\tilde{\alpha}(t)$ which preserves $\tilde{\Omega}$ is strongly continuous and - writing it as $e^{-i \tilde{H} t}-$ satisfies $\tilde{\varrho}\left(\mathfrak{Q}^{R}\right) \subset \mathscr{D}\left(e^{-\beta \tilde{\boldsymbol{H}} / 2}\right)$ with $e^{-\beta \tilde{H} / 2} \tilde{\varrho}(A) \tilde{\Omega}$ $=\tilde{\varrho}\left(l A^{*}\right) \tilde{\Omega} \forall A \in \mathfrak{Q}^{R}$.

Note that the restriction of such an $\tilde{\omega}^{\beta}$ to $\left(\mathfrak{Q}^{R}, \alpha(t)\right)$ [we adopt the convention $\alpha(t)=\tilde{\alpha}(t)\left\lceil_{\mathscr{A}^{R}}\right]$ is a KMS state in the usual sense. Moreover, (as explained in II) any dynamical system $(\mathfrak{U}, \alpha(t))$ may be viewed as the $\left(\mathfrak{H}^{R}, \alpha(t)\right)$ of some $(\tilde{\mathfrak{U}}, \tilde{\alpha}(t), l)$ and when so viewed, any KMS state on $(\mathfrak{H}, \alpha(t))$ arises as the restriction to $(\mathfrak{A}, \alpha(t))$ of a double KMS state on $(\tilde{\mathfrak{A}}, \tilde{\alpha}(t), \imath)$. The associated modular group $\Delta^{i t}$ is then $e^{-i \tilde{H} t \beta}$, and the modular involution arises as the unique complex conjugation $J$ satisfying $J \varrho(A) J=\tilde{\varrho}(\imath A), J \widetilde{\Omega}=\tilde{\Omega}$.

Theorem 1.3. For any double KMS state $\tilde{\omega}^{\beta}$ over a double dynamical system $(\widetilde{\mathfrak{A}}, \bar{\alpha}(t), \imath)$ (with GNS triple $(\tilde{\varrho}, \tilde{\mathscr{H}}, \widetilde{\Omega})$ and $\tilde{H}$ as in the above definition),

$$
\tilde{\varrho}\left(\mathfrak{Q}^{L}\right)^{\prime \prime}=\tilde{\varrho}\left(\mathfrak{A}^{R}\right)^{\prime} .
$$

If, in addition, the condition

$$
\forall \psi \in \mathscr{D}(\tilde{H}), \quad \tilde{H} \psi=0 \Rightarrow \psi=\lambda \tilde{\Omega}
$$

holds, then also

$$
\left.\tilde{\varrho}\left(\mathfrak{H}^{R}\right)^{\prime} \cap \varrho\left(\mathfrak{U}^{R}\right)^{\prime \prime}=\{\lambda \mathbb{1}\} \quad \text { (and similarly for } R \rightarrow L\right) .
$$

(iii) $\tilde{\varrho}(\tilde{\mathfrak{Q}})$ is irreducible, i.e. $\tilde{\omega}^{\beta}$ is pure. 1.3.

We say a double quantum dynamical system is (Bose) quasi-free if it arises [with $\tilde{\mathfrak{A}}$ the Weyl algebra over $(\tilde{D}, \tilde{\sigma}), \tilde{\alpha}(t) W(\Phi)=W(\tilde{\mathscr{T}}(t) \Phi), \imath(W(\Phi))=W(\mathscr{I} \Phi)]$, from a double classical linear dynamical system $(\tilde{D}, \tilde{\sigma}, \mathscr{T}(t), \mathscr{I})$.

Definition. This consists of a symplectic space $(\tilde{D}, \tilde{\sigma})$, a one-parameter symplectic group $\tilde{\mathscr{T}}(t)$ and an antisymplectic [i.e. $\left.\tilde{\sigma}\left(\mathscr{I} \Phi_{1}, \mathscr{I} \Phi_{2}\right)=-\tilde{\sigma}\left(\Phi_{1}, \Phi_{2}\right)\right]$ involution $\mathscr{I}$ on $(\tilde{D}, \tilde{\sigma})$ such that

(a) $[\tilde{\mathscr{T}}(t), \mathscr{I}]=0$,

(b) $\tilde{D}$ consists of the sum $D^{L}+D^{R}$ of two preferred independent subspaces such that

$$
\begin{gathered}
\tilde{\sigma}\left(\Phi^{L}, \Phi^{R}\right)=0 \quad \forall \Phi^{L} \in D^{L}, \quad \Phi^{R} \in D^{R}, \\
\tilde{T}(t): D^{L} \rightarrow D^{L}, \quad D^{R} \rightarrow D^{R}, \\
\left.\mathscr{I} D^{L}=D^{R} \quad \text { (and } \mathscr{I} D^{R}=D^{L}\right) .
\end{gathered}
$$

4 I.e. $l\left(A^{*}\right)=(\imath(A))^{*}, l(A B)=\imath(A) \iota(B), \imath(A+B)=\imath(A)+\imath(B), \imath(c A)=\bar{c} \iota(A)$ 
One may obtain a double KMS state $\tilde{\omega}^{\beta}$ over such an $(\tilde{\mathfrak{I}}, \tilde{\alpha}(t), l)$ by defining $\tilde{\omega}^{\beta}(W(\Phi))=\exp \left(-\frac{1}{2}\left\|\widetilde{K}^{\beta} \Phi\right\|_{\tilde{h}}^{2}\right)$, where $\left(\tilde{K}^{\beta}, \widetilde{h}\right)$ belong to a double KMS one-particle structure $\left(\tilde{K}^{\beta}, \tilde{h}, e^{-i \hbar h t}, j\right)$ over $(\tilde{D}, \tilde{\sigma}, \tilde{\mathscr{T}}(t), \mathscr{I})$.

Definition. This consists of a complex Hilbert space $\tilde{h}$; a real-linear map $\widetilde{K}^{\beta}: \widetilde{D} \rightarrow \widetilde{h}$ satisfying $2 \operatorname{Im}\left\langle\widetilde{K}^{\beta} \Phi_{1} \mid \widetilde{K}^{\beta} \Phi_{2}\right\rangle=\tilde{\sigma}\left(\Phi_{1}, \Phi_{2}\right)$ such that $\widetilde{K}^{\beta} D^{R}+i \widetilde{K}^{\beta} D^{R}$ is dense in $h$ (and similarly for $R \rightarrow L$ ); a strongly continuous one-parameter group $e^{-i \hbar t}$ on $\tilde{h}$ such that $\tilde{h}$ has no zero eigenvalues and $\widetilde{K}^{\beta} \tilde{\mathscr{T}}(t)=e^{-i \tilde{h} t} \widetilde{K}^{\beta}$, and a complex conjugation $j$ on $\widetilde{h}$ such that $\widetilde{K}^{\beta} D^{R}+i \widetilde{K}^{\beta} D^{R} C \mathscr{D}\left(e^{-\beta \tilde{h} / 2}\right)$ and $e^{-\beta \tilde{h} / 2} x=-j x \forall x \in \widetilde{K}^{\beta} D^{R}$ (and similarly with $R \rightarrow L$ and $\tilde{h} \rightarrow-\tilde{h}$ ).

Note. By Theorem 2 in II, it is a consequence of this definition that ran $\tilde{K}^{\beta}$ is dense in $\widetilde{h}$. Finally, corresponding to Theorem 1.1, we have:

Theorem 1.4. Given a double linear dynamical system $(\tilde{D}, \tilde{\sigma}, \tilde{\mathscr{T}}(t), \mathscr{I})$ for which are given two double KMS one-particle structures $\left(\widetilde{K}_{i}^{\beta}, \widetilde{h}_{i}, \exp \left(-i \widetilde{h_{i}} t\right), j_{i}\right), i=1,2$, for some given $0<\beta<\infty$, then there exists a unique unitary $U: \widetilde{h}_{1} \rightarrow \widetilde{h}_{2}$ such that

(a) $U \tilde{K}_{1}^{\beta}=\widetilde{K}_{2}^{\beta}$ on $\tilde{D}$,

(b) $U \exp \left(-i \tilde{h_{1}} t\right)=\exp \left(-i \tilde{h}_{2} t\right) U$ on $\tilde{h}_{1}$,

(c) $U j_{1}=j_{2} U$ on $\tilde{h}_{1}$.

1.4 .

Let $(\tilde{D}, \tilde{\sigma}, \tilde{T}(t), \mathscr{I})$ be a double classical linear dynamical system. Suppose $\left(D^{R}, \sigma, \mathscr{T}(t)\right)$ [where $\left.{ }^{5} \sigma=\tilde{\sigma} \uparrow_{D^{R}}, \mathscr{T}(t)=\tilde{\mathscr{T}}(t) \uparrow_{D^{R}}\right]$ admits a ground one particle structure $\left(K, h, e^{-i \hbar t}\right)$ (see Sect. 1.1) which satisfies the regularity condition $K D^{R}$ $C \mathscr{D}\left(h^{-1 / 2}\right)$. Then, if $C$ is any complex conjugation on $h$ such that $[C, h]=0$, the following construction gives a double KMS one-particle structure $\left(\tilde{K}^{\beta}, \widetilde{h}, e^{-i \tilde{h} t}, j\right)$ (unique up to equivalence by Theorem 1.4$)$ over $(\tilde{D}, \tilde{\sigma}, \tilde{T}(t), \mathscr{I})$ (cf. especially footnote 12 in II).

(1) Set $\tilde{h}=h \oplus h$.

(2) $\forall \Phi \in \tilde{D}$, let $\Phi=\Phi^{L}+\Phi^{R} ; \Phi^{L} \in D^{L}, \Phi^{R} \in D^{R}$, and set

$$
\tilde{K}^{\beta} \Phi=\left(\begin{array}{cc}
\cosh Z^{\beta} & \sinh Z^{\beta} C \\
\sinh Z^{\beta} C & \cosh Z^{\beta}
\end{array}\right)\left(\begin{array}{c}
-C K \mathscr{I} \Phi^{L} \\
K \Phi^{R}
\end{array}\right),
$$

where $Z^{\beta}$ is defined implicitly by

$$
\tanh Z^{\beta}=e^{-\beta h / 2} .
$$

[It is not difficult to see that the regularity condition $K D^{R} C \mathscr{D}\left(h^{-1 / 2}\right)$ suffices for $K D^{R} C \mathscr{D}\left(\cosh Z^{\beta}\right), K D^{R} C \mathscr{D}\left(\sinh Z^{\beta}\right)$ here. The details are given in Sect. A2 of I.]

(3) Set

$$
\exp (-i \tilde{h t})=\left(\begin{array}{cc}
e^{i h t} & 0 \\
0 & e^{-i h t}
\end{array}\right)
$$

(4) Set

$$
j=\left(\begin{array}{rr}
0 & -C \\
-C & 0
\end{array}\right)
$$

5 In the sequel, we shall not always make such redefinitions explicit 
Note that the resulting double KMS state

$$
\tilde{\omega}^{\beta}(W(\Phi))=\exp \left(-\frac{1}{2}\left\|\tilde{K}^{\beta} \Phi\right\|_{\tilde{n}}^{2}\right)
$$

on the quasi-free quantum dynamical system $(\tilde{\mathfrak{A}}, \tilde{\alpha}(t), \imath)$ arising (see Sect. 1.3) from $(\tilde{D}, \tilde{\sigma}, \tilde{T}(t), \mathscr{I})$ is given explicitly by the formula - splitting $\Phi$ as $\Phi^{L}+\Phi^{R} ; \Phi^{L} \in D^{L}$, $\Phi^{R} \in D^{R}$

$$
\begin{aligned}
\tilde{\omega}^{\beta}(W(\Phi))= & \exp \left\{-\frac{1}{2}\left\langle K \mathscr{I} \Phi^{L} \mid \operatorname{coth}(\beta h / 2) K \mathscr{I} \Phi^{L}\right\rangle\right. \\
& \left.-\frac{1}{2}\left\langle K \Phi^{R} \mid \operatorname{coth}(\beta h / 2) K \Phi^{R}\right\rangle+\operatorname{Re}\left\langle K \mathscr{I} \Phi^{L} \mid \operatorname{cosech}(\beta h / 2) K \Phi^{R}\right\rangle\right\} .
\end{aligned}
$$

The expressions in the exponent here are to be interpreted in the sense of quadratic forms, the condition $K D^{R} C \mathscr{D}\left(h^{-1 / 2}\right)$ again sufficing for $K D^{R} C Q(\operatorname{coth}(\beta h / 2)), K D^{R}$ $C Q(\operatorname{cosech}(\beta h / 2))$.

Note that in the corresponding (GNS) representation, $W(\Phi) \mapsto W^{\mathscr{F}}\left(\tilde{K}^{\beta} \Phi\right)$ on $\mathscr{F}(h) \otimes \mathscr{F}(h)$, the modular automorphism group is given by

$$
\Delta^{i t / \beta}=\Gamma\left(e^{-i \tilde{h}}\right)=\exp [-i t(-d \Gamma(h) \otimes \mathbb{1}+\mathbb{1} \otimes d \Gamma(h))],
$$

and the modular involution by

$$
J(\psi \otimes \varphi)=\Gamma(-C) \varphi \otimes \Gamma(-C) \psi .
$$

1.5.

Finally, we state a simple criterion for a von-Neumann algebra which is a factor to be of type $I I I_{1}$ in the sense of Connes [22]. It constitutes a very special case of the conditions stated in [22] but is adequate for our purpose.

Theorem 1.5. Let $\mathfrak{A}$ (acting on a Hilbert space $\mathscr{H}$ ) be a factor. Let $\Omega$ be a cyclic and separating vector for $\mathfrak{A}$ and $\Delta^{\text {it }}$ the corresponding modular group. If

(i) $\forall \psi \in \mathscr{H}, \Delta^{i t} \psi=\psi \Rightarrow \psi=\lambda \Omega$ and

(ii) the spectrum $\operatorname{sp}(\Delta)=[0, \infty)$, then $\mathfrak{A}$ is of type $I I I_{1}$.

\section{The Minkowski Vacuum}

We begin by constructing the algebra for the free Klein-Gordon equation in flat spacetime $\left(\mathscr{M} \approx \mathbb{R}^{4}, \eta\right)(\eta=\operatorname{diag}(1,-1,-1,-1))$

$$
\left(\square+m^{2}\right) \phi=0 \quad(m>0)
$$

in a suitable notation. We shall use coordinates $(T, X, \xi)$, where $\xi$ stands for $(Y, Z) \in \mathbb{R}^{2}$. [In Sect. A2 we shall consider generalizations without $\xi\left(\mathscr{M} \approx \mathbb{R}^{2}\right)$ or with $\xi$ standing for the last $d-2$ coordinates of an $\mathscr{M} \approx \mathbb{R}^{d}$ or for an element of $\mathbb{S}^{2}$ in the case $\mathscr{M} \approx \mathbb{R}^{2} \times \mathbb{S}^{2}$.]

Given a solution $\phi$ of (2.1) which is $C^{\infty}$ and has compact support on Cauchy surfaces, we define time-zero Cauchy data $f=\phi \uparrow_{T=0}, p=\phi \uparrow_{T=0}$. We use the abbreviation $D$ for the space $C_{0}^{\infty}(\mathscr{C}) \times C_{0}^{\infty}(\mathscr{C})$ of such data where by $\mathscr{C}\left(\cong \mathbb{R}^{3}\right)$ we denote the initial $T=0$ manifold. Denoting the right $(X>0)$ and left $(X<0)$ parts of $\mathscr{C}$ by $\mathscr{C}^{R}, \mathscr{C}^{L}$, we also assign the symbols $D^{R}, D^{L}$ to the subspaces $C_{0}^{\infty}\left(\mathscr{C}^{R}\right)$ $\times C_{0}^{\infty}\left(\mathscr{C}^{R}\right)$ and $C_{0}^{\infty}\left(\mathscr{C}^{L}\right) \times C_{0}^{\infty}\left(\mathscr{C}^{L}\right)$ of $D$ and finally denote by $\tilde{D}\left(=D^{L} \oplus D^{R}\right)$ the 
subspace $C_{0}^{\infty}\left(\mathscr{C}^{L} \cup \mathscr{C}^{R}\right) \times C_{0}^{\infty}\left(\mathscr{C}^{L} \cup \mathscr{C}^{R}\right)$ of $D$. We shall often denote an element $(f, p)$ of one of these spaces by the single symbol $\Phi$. Defining the symplectic form $\sigma$ over $D$ by

$$
\sigma\left(\Phi_{1}, \Phi_{2}\right)=\int_{\mathscr{C}}\left(f_{1} p_{2}-p_{1} f_{2}\right) d X d^{2} \xi
$$

$\left(d^{2} \xi\right.$ : usual volume element on $\mathbb{R}^{2}$ ), we construct the Weyl algebra $\mathfrak{A}$ generated by elements $W(\Phi), \Phi \in D$ satisfying

$$
W\left(\Phi_{1}\right) W\left(\Phi_{2}\right)=\exp \left(-\frac{i}{2} \sigma\left(\Phi_{1}, \Phi_{2}\right)\right) W\left(\Phi_{1}+\Phi_{2}\right) .
$$

We may regard $\mathfrak{A}$ as the $C^{*}$ algebra for a quantum solution $\hat{\phi}$ of (2.1) by defining $\exp \left(i \int_{\mathscr{M}} \hat{\phi} F d T d X d^{2} \xi\right), F \in C_{0}^{\infty}(\mathscr{M})$ to be $W(\Phi)$, where $\Phi$ are the time-zero Cauchy data of the solution $\Delta * F$, where $\Delta$ is the usual (advanced minus retarded) fundamental (distributional) solution of (2.1). By the causal support properties of $\Delta, \Phi$ will belong to $D^{R}$ whenever $F$ is supported in the right wedge $\mathscr{R} \approx\left\{(T, X, \xi) \in \mathbb{R}^{4}: X>|T|\right\}$ of Minkowski space, and we therefore define the right wedge subalgebra $\mathfrak{A}^{R}$ of $\mathfrak{A}$ to be that generated by $\left\{W(\Phi): \Phi \in D^{R}\right\}$. Similarly, we define the left wedge $\left(\mathscr{L} \approx\left\{(T, X, \xi) \in \mathbb{R}^{4}: X<|T|\right\}\right)$ subalgebra $\mathfrak{A}^{L} \subset \mathfrak{A}$ generated by $\left\{W(\Phi): \Phi \in D^{L}\right\}$ and the double-wedge $(\mathscr{L} \cup \mathscr{R})$ subalgebra $\tilde{\mathfrak{A}} \subset \mathfrak{A}$ generated by $\{W(\Phi): \Phi \in \tilde{D}\}$.

Next, we define some symplectic (antisymplectic) operators on $D$. First, we define the one-parameter symplectic group $\mathscr{T}\left(T^{\prime}\right)$ on $D$ to be the maps on timezero Cauchy data which correspond to the time-translations $\phi \mapsto \phi_{T^{\prime}}, \phi_{T^{\prime}}(T, X, \xi)$ $=\phi\left(T+T^{\prime}, X, \xi\right)$ on classical solutions. $(D, \sigma, \mathscr{T}(T))$ is then a classical linear dynamical system in the sense of Sect. 1.1.

Similarly, we define the one-parameter (symplectic) group $\mathscr{V}(t)$ on $D$ corresponding to the Lorentz boosts:

$$
\phi \mapsto \phi_{t}, \quad \phi_{t}(T, X, \xi)=\phi(\Lambda(t)(T, X), \xi),
$$

where

$$
\Lambda(t)\left(\begin{array}{l}
T \\
X
\end{array}\right)=\left(\begin{array}{ll}
\cosh t & \sinh t \\
\sinh t & \cosh t
\end{array}\right)\left(\begin{array}{l}
T \\
X
\end{array}\right) .
$$

Using the notation $f(X, \xi)=f(-X, \xi)$ etc. we also define the antisymplectic involution $\mathscr{I}(f, p)=(\tilde{f},-\breve{p})$ corresponding to the wedge-reflection map $\phi \rightarrow \phi_{r}$, $\phi_{r}(T, X, \xi)=\phi(-T,-X, \xi)$. Note that $\mathscr{V}(t): \widetilde{D} \rightarrow \tilde{D}$ with $D^{R} \rightarrow D^{R}, D^{L} \rightarrow D^{L}$, while $\mathscr{I}$ commutes with $\mathscr{V}(t)$ and maps $D^{R} \rightarrow D^{L}, D^{L} \rightarrow D^{R}$. Thus $(\tilde{D}, \sigma, \mathscr{V}(t), \mathscr{I})$ is a double linear classical system in the sense of Sect. 1.3.

Turning to the quantum theory, we define the dynamical $\beta(T)$ on $\mathfrak{A}$ by automorphisms

$$
\beta(T) W(\Phi)=W(\mathscr{T}(T) \Phi),
$$

so that $(\mathfrak{A}, \beta(T))$ is the usual quantum dynamical system (see Sect. 1.1) representing ordinary time evolution. 
We also define automorphisms $\tilde{\alpha}(t)$ by

$$
\tilde{\alpha}(t) W(\Phi)=W(\mathscr{V}(t) \Phi),
$$

and the involutary antiautomorphism $\imath$ by

$$
l(W(\Phi))=W(\mathscr{I} \Phi) .
$$

Clearly, $\tilde{\alpha}(t)$ inherits the properties $\tilde{\alpha}(t): \tilde{\mathfrak{A}} \rightarrow \tilde{\mathfrak{A}}$, with $\mathfrak{U}^{R} \rightarrow \mathfrak{U}^{R}, \mathfrak{U}^{L} \rightarrow \mathfrak{U}^{L}$, while $\imath$ commutes with $\tilde{\alpha}(t)$ and maps $\tilde{\mathfrak{A}} \rightarrow \tilde{\mathfrak{U}}$ with $\mathfrak{U}^{R} \rightarrow \mathfrak{U}^{L}, \mathfrak{U}^{L} \rightarrow \mathfrak{A}^{R}$, so that $(\tilde{\mathfrak{A}}, \tilde{\alpha}(t), \imath)$ is a double quantum dynamical system in the sense of Sect. 1.2.

We may now begin to discuss the relation between the usual ("Minkowski") quantization and the Fulling quantization. The usual Minkowski vacuum state $\omega_{0}$ may be specified by the generating functional

$$
\omega_{0}(W(\Phi))=\exp \left(-\frac{1}{2}\left\|k_{0} \Phi\right\|_{k_{0}}^{2}\right),
$$

where we take as one-particle Hilbert space $h_{0}=L_{\mathbb{C}}^{2}\left(\mathbb{R}^{3}\right)$, and $k_{0} \Phi=2^{-1 / 2}\left(\mu^{1 / 2} f+i \mu^{-1 / 2} p\right)$ with $\mu=\left(m^{2}-\nabla^{2}\right)^{1 / 2} \cdot \omega_{0}$ is designed to be a ground state for $(\mathfrak{A}, \beta(T))$. From the point of view of Sect. 1, this follows because $\left(k_{0}, h_{0}, \exp \left(-i h_{0} T\right)\right)\left(h_{0}=\mu\right)$ is a ground one-particle structure for the linear dynamical system $(D, \sigma, \mathscr{T}(T))$. In fact, in the corresponding (GNS) representation, $\varrho_{0}(W(\Phi))=W^{\mathscr{F}}\left(k_{0} \Phi\right)$ on $\mathscr{F}\left(h_{0}\right)$ and $\beta(T)$ is implemented by the positive energy unitary group $\Gamma\left(\exp \left(-i h_{0} T\right)\right)$. More importantly for us here, $\tilde{\alpha}(t)$ is implemented in the representation $\varrho_{0}$ by the unitary group $\Gamma\left(\exp \left(-i \kappa_{0} t\right)\right)$, where $\exp \left(-i \kappa_{0} t\right)$ is the usual one-particle implementor of Lorentz boosts (given explicitly in Sect. A1) characterized by $\exp \left(-i \kappa_{0} t\right) k_{0}=k_{0} \mathscr{V}(t)$. Finally, $l$ is implemented by the antiunitary $\Gamma\left(j_{0}\right)$, where $j_{0} \chi=C_{0} \check{\chi}$, and where $C_{0}$ (which represents ordinary one-particle time-reversal) is the natural complex conjugation on our $L_{\mathbb{C}}^{2}\left(\mathbb{R}^{3}\right)$ realization of $h_{0}$ and $\check{\chi}(X, \xi)=\chi(-X, \xi)$.

We now consider the restriction of $\omega_{0}$ to the double-wedge subalgebra $\tilde{\mathfrak{A}}$ of $\mathfrak{A}$. By an argument which is also valid for non-linear field theories, it follows from the Reeh-Schlieder theorem [specifically, from the fact that the Fock vacuum $\Omega^{\mathscr{F}}$ is cyclic for $\varrho_{0}\left(\mathfrak{U}^{R}\right)$ - see Sect. A1] and the Bisognano-Wichmann theorem [specifically $\varrho_{0}\left(\mathfrak{U}^{R}\right) \Omega \subset \mathscr{D}\left(\exp \left(-\pi d \Gamma\left(\kappa_{0}\right)\right)\right)$ and

$$
\exp \left(-\pi d \Gamma\left(\kappa_{0}\right)\right) \varrho_{0}(A) \Omega=\Gamma\left(j_{0}\right) \varrho_{0}\left(A^{*}\right) \Omega^{\mathscr{F}} \quad \forall A \in \mathfrak{U}^{R}
$$

- see Sect. A1] that $\omega_{0}$ on (say) $\mathfrak{I}^{R}$ is a KMS state for $\beta=2 \pi$ with respect to the evolution $\tilde{\alpha}(t)$. [In fact, it follows immediately from the above parenthetical remarks that, in the language of Sect. 1.2, $\omega_{0}$ is a double KMS state for $\beta=2 \pi$ on the double quantum dynamical system $(\tilde{\mathfrak{I}}, \tilde{\alpha}(t), \imath)$.

Our goal now is to give an alternative construction for $\omega_{0}$ on $\tilde{\mathfrak{A}}$ in terms of objects which are intrinsic to the double wedge. For this purpose, we turn to the Fulling quantization. In Sect. 4, we shall construct a regular ground one-particle structure $\left(k_{F}, h_{F}, \exp \left(-i h_{F} t\right)\right)$ for the linear dynamical system $\left(D^{R}, \sigma, \mathscr{V}(t)\right)$ (cf. Sects. 1.1, 1.4). Given this structure, we may construct for any $\beta>0$ the double KMS state $\tilde{\omega}_{F}^{\beta}$ over $(\tilde{\mathfrak{U}}, \tilde{\alpha}(t), \imath)$ by setting (cf. Sect. 1.4)

$$
\tilde{\omega}_{F}^{\beta}(W(\Phi))=\exp \left(-\frac{1}{2}\left\|\tilde{k}_{F}^{\beta} \Phi\right\|_{h_{F} \oplus h_{F}}^{2}\right) \quad \forall \Phi \in \tilde{D},
$$


where, writing $\Phi \in \tilde{D}$ as $\Phi^{L}+\Phi^{R} ; \Phi^{L} \in D^{L}, \Phi^{R} \in D^{R}$,

$$
\widetilde{k}_{F}^{\beta} \Phi=\left(\begin{array}{cc}
\cosh Z^{\beta} & \sinh Z^{\beta} C_{F} \\
\sinh Z^{\beta} C_{F} & \cosh Z^{\beta}
\end{array}\right)\left(\begin{array}{r}
\stackrel{-}{k}_{F}\left(\check{f}^{L}, \check{p}^{L}\right) \\
k_{F}\left(f^{R}, p^{R}\right)
\end{array}\right),
$$

where

$$
\tanh Z^{\beta}=\exp \left(-\beta h_{F} / 2\right),
$$

and for the complex conjugation $C_{F}$ we take (say) one-particle time reversal (i.e. the natural complex conjugation on the $L^{2}$ version of $h$ given in Sect. 4) which satisfies $C_{F} k_{F}\left(f^{R}, p^{R}\right)=k_{F}\left(f^{R},-p^{R}\right)$. Explicitly,

$$
\begin{aligned}
\tilde{\omega}_{F}^{\beta}(W(\Phi))= & \exp \left\{-\frac{1}{2}\left\langle k_{F}\left(\check{f}^{L}, \check{p}^{L}\right) \mid \operatorname{coth}\left(\frac{\beta h_{F}}{2}\right) k_{F}\left(\check{f}^{L}, \check{p}^{L}\right)\right\rangle\right. \\
& -\frac{1}{2}\left\langle k_{F}\left(f^{R}, p^{R}\right) \mid \operatorname{coth}\left(\frac{\beta h_{F}}{2}\right) k_{F}\left(f^{R}, p^{R}\right)\right\rangle \\
& \left.+\operatorname{Re}\left\langle k_{F}\left(\check{f}^{L},-\check{p}^{L}\right) \mid \operatorname{cosech}\left(\frac{\beta h_{F}}{2}\right) k_{F}\left(f^{R}, p^{R}\right)\right\rangle\right\}
\end{aligned}
$$

(with expressions in the exponent interpreted in the sense of quadratic forms - cf. Sect. 1.4).

Note that it is permissible to set $\beta=\infty$ in (2.12) (corresponding to zero temperature) and if one does so, one obtains a product state on $\mathfrak{A}^{L} \otimes \mathfrak{U}^{R}$ whose restriction to $\mathfrak{A}^{\boldsymbol{R}}$, say, is the Fulling vacuum state [2]

$$
\omega_{F}(W(\Phi))=\exp \left(-\frac{1}{2}\left\|k_{F} \Phi\right\|_{h_{F}}^{2}\right)
$$

which is a ground state for $\left(\mathfrak{U}^{R}, \alpha(t)\right)$.

Since, for linear systems, one does not expect phase transitions (apart from "Bose condensation" which is clearly absent for the states considered here ${ }^{6}$ ) one expects that (restricted to $\mathfrak{U}^{R}$ ) $\tilde{\omega}_{F}^{2 \pi}$ must equal $\omega_{0}$ since both are KMS states over $\left(\mathfrak{I}^{R}, \alpha(t)\right)$. [In fact, both are double KMS states over $(\tilde{\mathfrak{A}}, \tilde{\alpha}(t), \imath)-$ see Sect. 1.2 for the same $\beta$.] That this is indeed the case (and extends to $\tilde{\mathfrak{I}}$ ) is the content of the following theorem.

Theorem 2.1. On $\tilde{\mathfrak{A}}, \omega_{0}=\tilde{\omega}_{F}^{2 \pi}$.

Proof. Defining $\tilde{h}_{F}=h_{F} \oplus h_{F}, \tilde{h}_{F}=h_{F} \oplus-h_{F}$ and $j_{F}\left(\chi_{1} \oplus \chi_{2}\right)=-C_{F} \chi_{2} \oplus-C_{F} \chi_{1}$, observe that $\left(k_{0}, h_{0}, \exp \left(-i \kappa_{0} t\right), j_{0}\right)$ and $\left(\widetilde{k}_{F}^{2 \pi}, \widetilde{h}_{F}, \exp \left(-i \widetilde{h}_{F} t\right), j_{F}\right)$ are each double KMS one-particle structures (Sect. 1.3) over $(\tilde{D}, \sigma, \mathscr{V}(t), \mathscr{I})$ for $\beta=2 \pi$. For the case of $\left(\widetilde{k}_{F}^{2 \pi}, \tilde{h}_{F}, \exp \left(-i \kappa_{F} t\right), j_{F}\right)$ this is a special case of the construction given in Sect. 1.4. For the case of $\left(k_{0}, h_{0}, \exp \left(-i \kappa_{0} t\right), j_{0}\right)$, this is the content of the pre-Reeh-

6 We refer to the possibility that two KMS states (at the same $\beta$ ) $\omega_{1}, \omega_{2}$ could be related by $\omega_{2}(W(\Phi))=\omega_{1}(W(\Phi)) e^{i \chi(\Phi)}$ for some non-zero $\mathscr{V}(t)$-invariant linear functional $\chi$ on the $\Phi$ 's. This is irrelevant for the states considered here because the exponents in (2.8) and (2.13) are each purely quadratic with no linear term in $\Phi$ 
Schlieder and pre-Bisognano-Wichmann theorems which are proved in Sect. A1 together with the elementary fact that $\kappa_{0}$ has no zero eigenvalues. The equality of $\omega_{0}$ and $\tilde{\omega}_{F}^{2 \pi}$ on $\widetilde{\mathfrak{A}}$ then follows by Theorem 1.4.

\section{The Hartle-Hawking State}

We now turn to the covariant Klein-Gordon equation

$$
\left(\square_{g}+m^{2}\right) \phi=0
$$

(now for any mass $m \geqq 0$ ) on the Kruskal spacetime ${ }^{7}\left(\mathscr{M} \approx \mathbb{R}^{2} \times \mathbb{S}^{2}, g\right)$ (see Fig. 1) of mass $M$ with metric [23]

$$
g=32 M^{3} r^{-1} e^{-r / 2 M}\left(d T^{2}-d X^{2}\right)-r^{2} d \Omega_{\xi}^{2},
$$

where the coordinates $(T, X, \xi)\left(\xi \in \mathbb{S}^{2}\right)$ range over the region $T^{2}-X^{2}<1$ of $\mathbb{R}^{2} \times \mathbb{S}^{2}, d \Omega_{\xi}^{2}$ is the usual metric on $\mathbb{S}^{2}$, and the Schwarzschild $r$ is defined implicitly in terms of $T$ and $X$ by

$$
T^{2}-X^{2}=(1-r / 2 M) e^{r / 2 M} .
$$

We shall be particularly interested in the exterior Schwarzschild $(r>2 M)$ right and left wedge regions $\mathscr{R}(X>|T|)$ and $\mathscr{L}(X<|T|)$ and in the double wedge $\mathscr{R} \cup \mathscr{L}$.

As in Sect. 2, we shall work with the $T=0$ Cauchy surface $\mathscr{C}\left(\approx \mathbb{R}^{2} \times \mathbb{S}^{2}\right)$ and the $X>0$ and $X<0$ parts $\mathscr{C}^{\mathbb{R}}\left(\approx \mathbb{R}^{+} \times \mathbb{S}^{2}\right)$ and $\mathscr{C}^{L}\left(\approx \mathbb{R}^{-} \times \mathbb{S}^{2}\right)$ of $\mathscr{C}$ which are Cauchy surfaces, respectively for $\mathscr{R}$ and $\mathscr{L}$. Defining the corresponding space of Cauchy data $D=C_{0}^{\infty}(\mathscr{C}) \times C_{0}^{\infty}(\mathscr{C})$ [and denoting, by $D^{R}$ and $D^{L}$, the subspaces $C_{0}^{\infty}\left(\mathscr{C}^{R}\right) \times C_{0}^{\infty}\left(\mathscr{C}^{R}\right)$ and $C_{0}^{\infty}\left(\mathscr{C}^{L}\right) \times C_{0}^{\infty}\left(\mathscr{C}^{L}\right)$, and, by $\left.\tilde{D}, D^{L}+D^{R}\right]$ we state the

7 We shall sometimes use the same symbol in Sects. 2 and 3, for quantities which are analogous

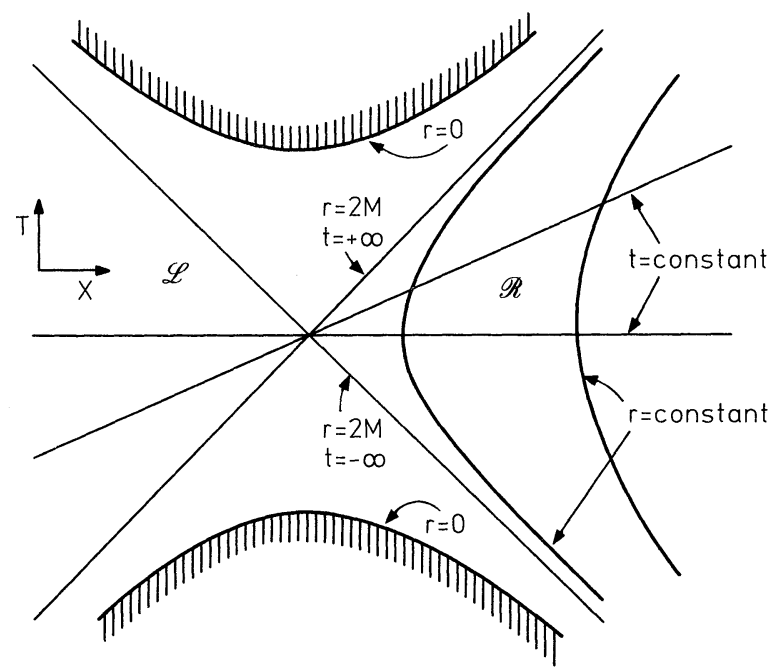

Fig. 1. The Kruskal extension of Schwarzschild spacetime 
Proposition 3.1 (Well-posedness of the Cauchy problem for (3.1)). Given Cauchy data $\Phi=(f, p) \in D$ there is a unique solution $\phi \in C^{\infty}(\mathscr{M})$ of (3.1) such that ${ }^{8}$ $r \phi \uparrow_{\mathscr{C}}=f, r \frac{\partial \phi}{\partial T} \uparrow_{\mathscr{C}}=p$. Moreover, the support of $\phi$ is in the domain of dependence [23] of the support of $\Phi$.

One may prove this by standard energy-estimate methods using the fact that $\mathscr{M}$ may be written (in the $C^{\infty}$ sense) as a product manifold $\approx \mathbb{R} \times \mathscr{C}$ with each $\{\tau\} \times \mathscr{C}$ Cauchy. Alternatively, note that this latter property implies [24] that $\mathscr{M}$ is globally hyperbolic and thus the result follows by the general Leray theory $[25$, 26]. See [14] for further discussion.

We introduce a symplectic form $\sigma$ on $D$ by integrating the conserved current $\left(\partial_{\mu}{ }^{\mu}=0\right)$ for a pair of solutions $\phi_{1}, \phi_{2}$

$$
j^{\mu}\left(\phi_{1}, \phi_{2}\right)=(-\operatorname{det} g)^{1 / 2} g^{\mu v}\left(\phi_{1} \partial_{v} \phi_{2}-\phi_{2} \partial_{v} \phi_{1}\right)
$$

over $\mathscr{C}$ to obtain

$$
\sigma\left(\Phi_{1}, \Phi_{2}\right)=\int_{\mathscr{C}}\left(f_{1} p_{2}-p_{1} f_{2}\right) d X d^{2} \xi
$$

(here $d^{2} \xi$ denotes the volume element on $\mathbb{S}^{2}$ ).

We then construct the Weyl algebra $\mathfrak{A}$ over $(D, \sigma)$ as before [cf. (2.3)]. $\mathfrak{A}$ may be regarded as the $C^{*}$ algebra for a quantum solution $\hat{\phi}$ of (3.1) by defining $\exp \left(i \int_{\mathscr{M}} \hat{\phi} F(-\operatorname{det} g)^{1 / 2} d T d X d^{2} \xi\right), F \in C_{0}^{\infty}(\mathscr{M})$ to be $W(\Phi)$, where $\Phi$ are the timezero Cauchy data of the solution $E F$, where $E$ is the classical fundamental (advanced minus retarded) solution of (3.1) viewed as an operator from $C_{0}^{\infty}(\mathscr{M}) \rightarrow C^{\infty}(\mathscr{M})($ see $[13,14])$. By the causal support properties of $E, \Phi$ will belong to $D^{R}$ whenever $F$ is supported in $\mathscr{R}$, and we therefore define the right wedge subalgebra $\mathfrak{U}^{R}$ of $\mathfrak{A}$ to be that generated by $\left\{W(\Phi): \Phi \in D^{R}\right\}$. Similarly, we define the left wedge subalgebras $\mathfrak{A}^{L} \subset \mathfrak{A}$ generated by $\left\{W(\Phi): \Phi \in D^{L}\right\}$ and the double wedge subalgebra $\widetilde{\mathfrak{A}} \subset \mathfrak{A}$ generated by $\{W(\Phi): \Phi \in \widetilde{D}\}$.

Next, we define analogues to the symplectic group $\mathscr{V}(t)$ and the antisymplectic involution $\mathscr{I}$ of Sect. 2. (There is no analogue on Kruskal to the time-translational symmetry $T \rightarrow T+T^{\prime}$.) We define the one-parameter group $\mathscr{V}(t)$ on $D$ corresponding to the transformations $\phi \mapsto \phi_{t} ; \phi_{t}(T, X, \xi)=\phi(\Lambda(t)(T, X), \xi)$, where $\Lambda(t)$ is the one-parameter group of isometries given in Kruskal coordinates by

$$
\Lambda(t)\left(\begin{array}{c}
T \\
X
\end{array}\right)=\left(\begin{array}{ll}
\cosh (t / 4 M) & \sinh (t / 4 M) \\
\sinh (t / 4 M) & \cosh (t / 4 M)
\end{array}\right)\left(\begin{array}{c}
T \\
X
\end{array}\right) .
$$

(On $\mathscr{R}$, this coincides with the usual Schwarzschild time translations - see Sect. 4.) That $\mathscr{V}(t)$ maps $D \rightarrow D$ and preserves $\sigma$ is guaranteed by Theorem 3.1 and the

8 There is some freedom in the association $\phi \mapsto \Phi$ of Cauchy data to solutions. Other conventions are possible provided we consistently adjust $\sigma\left(\Phi_{1}, \Phi_{2}\right)$ [cf. (3.4) below] to correspond to $\int_{\mathscr{C}} j^{\mu}\left(\Phi_{1}, \Phi_{2}\right) \varepsilon_{\mu \nu \lambda \sigma} d x^{\nu} d x^{\lambda} d x^{\sigma}$ [and correspondingly change $\mathscr{V}(t)$ etc.]. The subsequent interpretation of the Weyl algebra is then assured to correspond to the standard canonical quantization. The convention chosen here makes $\sigma$ simple in Kruskal coordinates. We shall change convention $\left[\right.$ for $\left.\left(D^{R},\left.\sigma\right|_{D^{R}}\right)\right]$ in Sect. 4 when we choose different coordinates (on $\left.\mathscr{R}\right)$ 
conservation of $j^{\mu}\left(\phi_{1}, \phi_{2}\right)$. Using the notation $\check{h}(X, \xi)=h(-X, \xi)$, we also define the antisymplectic involution $\mathscr{I}(f, p)=(\breve{f},-\check{p})$ corresponding to the wedge-reflection map $\phi \mapsto \phi_{r} ; \phi_{r}(T, X, \xi)=\phi(-T,-X, \xi)$. Just as in Sect. 2, we have (again by Theorem 3.1) $\mathscr{V}(t): \tilde{D} \rightarrow \tilde{D}$ with $D^{R} \rightarrow D^{R}, D^{L} \rightarrow D^{L}$, while $\mathscr{I}$ commutes with $\mathscr{V}(t)$ and maps $\tilde{D} \rightarrow \tilde{D}$ with $D^{R} \rightarrow D^{L}, D^{L} \rightarrow D^{R}$, so that $(\tilde{D}, \sigma, \mathscr{V}(t), \mathscr{I})$ is a double classical linear dynamical system in the sense of Sect. 1.3.

Turning to the quantum theory, we define the automorphisms $\tilde{\alpha}(t)$ on $\mathfrak{A}$ by

$$
\tilde{\alpha}(t) W(\Phi)=W(\mathscr{V}(t) \Phi)
$$

and the involutary antiautomorphism $\imath$ by

$$
{ }_{\imath} W(\Phi)=W(\mathscr{I} \Phi),
$$

which clearly inherit the properties $\tilde{\alpha}(t): \widetilde{\mathfrak{U}} \rightarrow \tilde{\mathfrak{U}}$ with $\mathfrak{Q}^{R} \rightarrow \mathfrak{U}^{R}, \mathfrak{U}^{L} \rightarrow \mathfrak{U}^{L}$ while $\imath$ commutes with $\tilde{\alpha}(t)$ and maps $\tilde{\mathfrak{A}} \rightarrow \tilde{\mathfrak{U}}$ with $\mathfrak{A}^{R} \rightarrow \mathfrak{U}^{L}, \mathfrak{A}^{L} \rightarrow \mathfrak{U}^{R}$, so that $(\tilde{\mathfrak{U}}, \tilde{\alpha}(t), \imath)$ is a double quantum dynamical system in the sense of Sect. 1.2.

We now construct a state (the Hartle-Hawking state) $\omega_{H}$ on the double-wedge algebra $\tilde{\mathfrak{A}}$ of Kruskal which is in many ways analogous to the vacuum state $\omega_{0}$ on Minkowski space. Since there is no analogue of the time-translation group $\beta(T)$ of Minkowski space, we follow the strategy outlined in the introduction and construct $\omega_{H}$ as a double KMS state (for $\beta=8 \pi M$ ) over ( $\left.\tilde{\mathfrak{A}}, \tilde{\alpha}(t), \imath\right)$. For this purpose, we turn to the Boulware quantization. In Sect. 4, we shall construct a regular ground one-particle structure (see Sects. 1.1 and 1.4) $\left(k_{B}, h_{B}, \exp \left(-i h_{B} t\right)\right)$ for the linear dynamical system $\left(D^{R}, \sigma, \mathscr{V}(t)\right)$. We may then construct for any $\beta>0$ a state $\tilde{\omega}_{B}^{\beta}$ by replacing $F$ (for Fulling) by $B$ (for Boulware) in (2.9)-(2.12). We then define the Hartle-Hawking state $\omega_{H}$ to be $\tilde{\omega}_{B}^{8 \pi M}$. We may then immediately conclude

Theorem 3.2. (A) $\omega_{H}$ on $\widetilde{\mathfrak{A}}$ is a pure state [i.e. in the corresponding (GNS) Hilbert space representation $\varrho_{H}, \varrho_{H}(\tilde{\mathfrak{Q}})$ is irreducible $]$.

(B) $\Omega$ (the GNS vacuum) is cyclic for $\varrho_{H}\left(\mathfrak{A}^{R}\right), \varrho_{H}\left(\mathfrak{Q}^{L}\right)$ (Reeh-Schlieder property). have

Moreover, defining the von-Neumann algebras $\mathscr{A}_{L}=\varrho_{H}\left(\mathfrak{U}^{L}\right)^{\prime \prime}, \mathscr{A}_{R}=\varrho_{H}\left(\mathfrak{I}^{R}\right)^{\prime \prime}$ we

(C) $\mathscr{A}_{L}^{\prime}=\mathscr{A}_{R}$ (duality of wedge algebras).

(D) $\mathscr{A}_{L} \cap \mathscr{A}_{L}^{\prime}=\{\lambda \mathbb{1}\}, \mathscr{A}_{R} \cap \mathscr{A}_{R}^{\prime}=\{\lambda \mathbb{1}\}$ (i.e. $\mathscr{A}_{L}$ and $\mathscr{A}_{R}$ are factors).

(E) The factors $\mathscr{A}_{L}, \mathscr{A}_{R}$ are of type III ${ }_{1}$ in the classification of Connes.

Proof. Since $\omega_{H}$ arises from a double KMS one-particle structure $\left(\tilde{k}_{B}^{8 \pi M}, \tilde{h}_{B}\right.$, $\exp \left(-i \tilde{h}_{B} t\right), j_{B}$ ) (cf. the reference to Sect. 1.4 in the proof of Theorem 2.1) over $(\tilde{D}, \sigma, \mathscr{V}(t), \mathscr{I})$, it is automatically (see Sect. 1.3) a double KMS state over $(\tilde{\mathfrak{A}}, \tilde{\alpha}(t), \imath)$ and hence (B) holds immediately. It also satisfies Condition (a) of Theorem 1.3 since, because $h_{B}$ and hence $\widetilde{h}_{B}$ have no zero eigenvalues $d \Gamma\left(\widetilde{h}_{B}\right) \psi=\psi \Rightarrow \psi=\Omega$. (A), (C), (D) above then correspond to parts (iii), (i), (ii), respectively of Theorem 1.3.

For part (E), it suffices by Theorem 1.5 [we again use Condition (a)] to show $\operatorname{sp}(\Delta)=[0, \infty)$. By Sect. 1.4, $\Delta=e^{-\beta \tilde{H}}$, where $\beta=8 \pi M$ and $\tilde{H}=-d \Gamma\left(h_{B}\right) \otimes \mathbb{1}$ $+\mathbb{1} \otimes d \Gamma\left(h_{B}\right)$. It thus suffices to show that $\operatorname{sp}\left(h_{B}\right)=[0, \infty)\left[\right.$ for then $\operatorname{sp}\left(d \Gamma\left(h_{B}\right)\right)$ $=[0, \infty)$ and $\operatorname{sp}(\tilde{H})=(-\infty, \infty)]$. This fact (which incidentially entails the absence of a mass gap for the Boulware quantization) will be established in Sect. A3. 
We briefly discuss the extent to which this theorem might continue to hold for non-linear field theories. It seems reasonable to expect that one will continue to be able to define a double-wedge quantum dynamical system $(\widetilde{\mathfrak{A}}, \tilde{\alpha}(t), l)$ in such cases, and that the concept of Hartle-Hawking state will still make sense. Whatever else it may be, $\omega_{H}$ will presumably still be a double KMS state (with $\beta=8 \pi M$ ) over $(\widetilde{\mathfrak{A}}, \tilde{\alpha}(t), l)$ in the sense of Sect. 1.2 and therefore part (C) (duality) of Theorem 3.2 should remain true. If "uniqueness of the vacuum" [i.e. Condition (a) of Theorem 1.3] also holds then we shall also have parts (A) and (D). The situation for part (E) is less clear, since for part (E), the present proof relies heavily on special features of the quasi-free case.

\section{Construction of (Regular) Fulling}

\section{and Boulware Ground One-Particle Structures}

In this section, we show that the $\left(D^{R}, \sigma, \mathscr{V}(t)\right)$ of each of our equations [i.e. (2.1) for $m>0$, and (3.1) for $m \geqq 0]$ admit a regular ground one-particle structure (see Sects. 1.1 and 1.4) $\left(k, h, e^{-i h t}\right)$. (We shall treat both cases together and thus drop the suffices $F$ and $B$.)

It will be convenient to work in $(t, x, \xi)$ coordinates for $\mathscr{R}$. Here, $t$ and $x$ which range over $\mathbb{R}^{2}$ are defined by [23]

$$
T=e^{x / 4 M} \sinh (t / 4 M), \quad X=e^{x / 4 M} \cosh (t / 4 M) .
$$

In the Schwarzschild case, $x$ is the Regge-Wheeler radial coordinate - often called $r_{*}-$ and $t$ the Schwarzschild time. In the Minkowski case, we tacitly take $4 M=1 . \xi$ - which ranges over $\mathbb{R}^{2}$ in the Minkowski, and $\mathbf{S}^{2}$ in the Schwarzschild case - is unchanged. The transformations $\Lambda\left(t^{\prime}\right)$ of (2.4) and (3.5) then become $(t, x, \xi)$ $\rightarrow\left(t+t^{\prime}, x, \xi\right)$ and our equations each acquire the special first-order form (cf. Sect. 7 in [27])

$$
\left(\begin{array}{c}
\dot{u} \\
\ddot{u}
\end{array}\right)=-\mathbf{h}\left(\begin{array}{c}
u \\
\dot{u}
\end{array}\right) .
$$

Here, $u$ stands for $r \phi$ in the case of (3.1) and for $\phi$ in the case of (2.1),

$$
\mathbf{h}=\left(\begin{array}{rr}
0 & -1 \\
A & 0
\end{array}\right)
$$

where $A$ is given for (2.1) by

$$
A=-\frac{\partial^{2}}{\partial x^{2}}+e^{2 x}\left(m^{2}-\Delta_{\xi}\right)
$$

(with $\Delta_{\xi}=$ Laplacian on $\mathbb{R}^{2}$ ) and for (3.1) by

$$
A=-\frac{\partial^{2}}{\partial x^{2}}+\left(1-\frac{2 M}{r}\right)\left(\frac{2 M}{r^{3}}-\frac{\Delta_{\xi}}{r^{2}}+m^{2}\right)
$$

with $\Delta_{\xi}=$ Laplacian on $\mathbf{S}^{2}$ and $r$ the Schwarzschild radial coordinate [see (3.3)] which is given implicitly in terms of $x$ by

$$
x=r+2 M \ln (r / 2 M-1) .
$$


In Sect. 7 of [27], we constructed a ground one-particle structure for a class of equations similar to (4.2). We will follow a similar route here. However, the conditions assumed in [27] necessitated an $A$ [corresponding to (4.4)/(4.5) here] with a positive lower bound (i.e. $\left.\langle f \mid A f\rangle \geqq \varepsilon\langle f \mid f\rangle \forall f \in L^{2}\left(\mathscr{M}, d x d^{2} \xi\right)\right)$. This greatly simplified the construction and also gave as a consequence a mass gap for the quantum theory ${ }^{9}$ (i.e. $\langle\chi \mid h \chi\rangle \geqq \varepsilon^{\prime}\langle\chi \mid \chi\rangle \forall \chi \in h$ ). The $A$ 's of (4.4), (4.5) clearly have no positive lower bound. (The consequent absence of a mass gap was briefly mentioned by us in [28].) This will require us in what follows to deal with a number of new technical issues. For convenience, we relegate some of the necessary operator theory to Sect. A4.

The essential common feature of our $A$ 's [(4.4) and (4.5)] that we will use is that they each satisfy an estimate of the form

$$
\int_{\mathscr{C}^{R}} f A f d x d^{2} \xi \geqq \int_{\mathscr{C}^{R}} \alpha|f|^{2} d x d^{2} \xi \quad \forall f \in C_{0}^{\infty}\left(\mathscr{C}^{R}\right)
$$

for some strictly positive function $\alpha>0$. One may take, for (4.4) $\alpha=e^{2 x} m^{2}$ and for (4.5) $\alpha=(1-2 M / r)\left(2 M / r^{3}\right)$. Notice that this is the point at which we require $m>0$ for (2.1) (cf. Sect. A2) and that such a restriction is unnecessary for (3.1).

Corresponding to our choice of coordinates, we shall work with an equivalent (see footnote 6) version $(\hat{D}, \delta, \delta, \delta(t))$ of $\left(D^{R}, \sigma, \mathscr{V}(t)\right)$ by taking for $\hat{D}$ a copy of $D^{R}$ [in the chosen coordinates $\approx C_{0}^{\infty}\left(\mathbb{R} \times \mathbb{S}^{2}\right) \times C_{0}^{\infty}\left(\mathbb{R} \times \mathbb{S}^{2}\right)$ for (3.1) and $C_{0}^{\infty}\left(\mathbb{R}^{3}\right) \times C_{0}^{\infty}\left(\mathbb{R}^{3}\right)$ for (2.1)]; identifying an old $\Phi=(f, p) \in D^{R}$ with $\widetilde{\Phi}=(f, \widehat{p}) \in \tilde{D}$, where $\hat{p}=e^{x / 4 M} p$, so that $\delta$ defined by $\delta\left(\overleftarrow{\Phi}_{1}, \overleftarrow{\Phi}_{2}\right)=\sigma\left(\Phi_{1}, \Phi_{2}\right)$ becomes

$$
\overleftarrow{\sigma}\left(\overleftarrow{\Phi}_{1}, \overleftarrow{\Phi}_{2}\right)=\int_{\mathscr{G} R}\left(f_{1} \widehat{p}_{2}-\widehat{p}_{1} f_{2}\right) d x d^{2} \xi,
$$

and by defining $\mathscr{V}^{5}(t)$ by

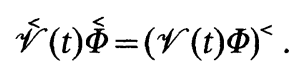

We know from Sects. 2 and 3 that $(4.1)$ is solved for initial data $(u(0), \dot{u}(0))$ $=(f, \grave{p}) \in \widehat{D}$ by

$$
\overleftarrow{\Phi}(t)=(u(t), \dot{u}(t))=\overleftarrow{\mathscr{V}}(t)(f, \widehat{p}),
$$

and in particular, we have by Proposition 3.1 for Schwarzschild (and an analogous more elementary result for Minkowski)

Proposition 4.1. Such a solution $\overleftarrow{\Phi}(t)$ (viewed as a pair of functions on $\mathscr{R}$ ) is $C^{\infty}$ and has compact support in any region of bounded t coordinate.

Next we note that, in addition to preserving $\delta, \mathscr{V}(t)$ also preserves the energy norm [that this is a norm follows e.g. from (4.7)] defined by

$$
\|\widehat{\Phi}\|_{\mathscr{A}}^{2}=\frac{1}{2} \int_{\mathscr{G} R}\left(f A f+\hat{p}^{2}\right) d x d^{2} \xi .
$$

We will now consider $\overleftarrow{\mathscr{V}}^{<}(t)$ as extended in the usual way to a one-parameter (orthogonal) group on the Hilbert space completion $\mathscr{A}$ of $D$ in this norm. By Eqs. (4.2), (4.10), and Proposition 4.1, we may conclude (on using the dominated convergence theorem and the mean value theorem in the appropriate expressions)

9 Note also that, in consequence of this, the regularity condition of Sects. 1.1 and 1.4 was automatically satisfied in the case of [27] 
that $\mathscr{V}^{\leftarrow}(t)$ is strongly continuous on $\mathscr{A}$ with strong derivative $-\mathbf{h}$ on $\overleftarrow{D}$. It follows by Proposition A4.1 that $\mathbf{h}$ is essentially skew-adjoint on $\bar{D} \subset \mathscr{A}$ with (using the symbol h now for the corresponding self-adjoint closure)

$$
\hat{\mathscr{V}}(t)=e^{-\mathbf{h} t}
$$

Further, Proposition A4.2 assures us that

$$
\mathbf{h}^{2}=-\left(\begin{array}{ll}
A & 0 \\
0 & A
\end{array}\right)
$$

is essentially self-adjoint on $\mathscr{D}_{C \mathscr{A}}$ and thus, restricting to the subspace of $\mathscr{A}$ generated by Cauchy data of form $(0, \widehat{p})$, we conclude (exactly as in [27], cf. [29])

Proposition 4.2. $A$ (as in (4.4) or (4.5)) is essentially self-adjoint on $C_{0}^{\infty}\left(\mathscr{C}^{R}\right)$ $C L^{2}\left(\mathscr{C}^{R}, d x d^{2} \xi\right)$.

By (4.7), $A$ is positive on $C_{0}^{\infty}\left(\mathscr{C}^{R}\right)$ and hence its closure (which we shall also denote by $A$ ) is a positive operator. In fact, it follows from (4.7), using Proposition A4.7 that

Proposition 4.3. $A$ is strictly positive.

Now restrict $e^{-\mathbf{h t}}$ from $\mathscr{A}$ to the dense invariant domain

$$
\mathscr{D}\left(A^{1 / 2}\right) \oplus\left(L^{2}\left(\mathscr{C}^{R}, d x d^{2} \xi\right) \subset \mathscr{A} .\right.
$$

This is a genuine restriction since $A$ (as already mentioned) has no positive lower bound (cf. [30, Sect. XI.10]). On this domain, it is straightforward to show that $e^{-\mathbf{h t}}$ restricts to

$$
\left(\begin{array}{cc}
\cos \left(A^{1 / 2} t\right) & A^{-1 / 2} \sin \left(A^{1 / 2} t\right) \\
-A^{1 / 2} \sin \left(A^{1 / 2} t\right) & \cos \left(A^{1 / 2} t\right)
\end{array}\right) .
$$

(In [16], we shall require and use the extension of this formula to all of $\mathscr{A}$ as given in [30, Sect. XI.10].) Also, the symplectic form defined by (4.8) clearly extends to this domain and continues to be preserved by $\mathscr{V}^{r}(t)$.

To construct a regular ground one-particle structure, we restrict further to the invariant domain $\mathscr{D}\left(A^{1 / 2}\right) \oplus \mathscr{D}\left(A^{-1 / 2}\right)$ and define the map

$$
\grave{k}:(f, \widehat{p}) \mapsto 2^{-1 / 2}\left(A^{1 / 4} f+i A^{-1 / 4} \widehat{p}\right)
$$

into the complex Hilbert space $L_{\mathbb{C}}^{2}\left(\mathscr{C}^{R}, d x d^{2} \xi\right)$. On this domain, $\npreceq$ clearly intertwines $e^{-\mathbf{h t}}$ with the strictly positive energy (Propositions 4.3 and A4.5) unitary group $\exp \left(-i A^{1 / 2} t\right)$ and is symplectic. In fact,

Proposition 4.4. $\left(\hat{k}, L_{\mathbb{C}}^{2}\left(\mathscr{C}^{R}, d x d^{2} \xi\right), e^{-i h t}\right), h=A^{1 / 2}$, is a regular ground one-particle structure over $\left(\mathscr{D}\left(A^{1 / 2}\right) \oplus \mathscr{D}\left(A^{-1 / 2}\right), \widehat{\sigma}, e^{-\mathrm{h} t}\right)$.

Proof. It only remains to show that $\operatorname{ran} k$ is dense and to check regularity. The former reduces to showing that $A^{1 / 4} \mathscr{D}\left(A^{1 / 2}\right)$ [and $A^{-1 / 4} \mathscr{D}\left(A^{-1 / 2}\right)$ - which by $A^{-1 / 4} \mathscr{D}\left(A^{-1 / 2}\right)=A^{1 / 4} A^{-1 / 2} \mathscr{D}\left(A^{-1 / 2}\right)=A^{1 / 4} \mathscr{D}\left(A^{1 / 2}\right)$ is the same thing] is dense in $L_{\mathbb{R}}^{2}\left(\mathscr{C}^{R}, d x d^{2} \xi\right)$. This follows by Propositions 4.3 and A4.3-A4.6. The regularity condition easily reduces to showing $A^{1 / 4} \mathscr{D}\left(A^{1 / 2}\right)\left(=A^{-1 / 4} \mathscr{D}\left(A^{-1 / 2}\right)\right) \subset \mathscr{D}\left(A^{-1 / 4}\right)$, which is obvious. 
The above proposition will be relevant for the discussion of scattering theory in [16] (see also Sects. A2 and A3). The result we require here is ${ }^{10}$

Theorem 4.5. $\mathscr{D}\left(A^{1 / 2}\right) \oplus \mathscr{D}\left(A^{-1 / 2}\right) \supset \grave{D}$ and the restriction of $\left(\stackrel{k}{k} L_{\mathbb{C}}^{2}\left(\mathscr{C}^{R}, d x d^{2} \xi\right), e^{-i h t}\right)$ to $\left(\bar{D}, \zeta, \mathscr{V}^{2}(t)\right)$ remains a regular ground one-particle structure.

Proof. To show $\bar{D} \subset \mathscr{D}\left(A^{1 / 2}\right) \oplus \mathscr{D}\left(A^{-1 / 2}\right)$ clearly amounts to showing (i) $C_{0}^{\infty}\left(\mathscr{C}^{R}\right)$ $C \mathscr{D}\left(A^{1 / 2}\right)$ and (ii) $C_{0}^{\infty}(\mathscr{C}) \subset \mathscr{D}\left(A^{-1 / 2}\right)$. (i) is obvious. For (ii), use again the estimate (4.7) and Proposition A4.9. Finally, we need to show that $\operatorname{ran} k$ remains dense, i.e. that (i) $A^{1 / 4} C_{0}^{\infty}\left(\mathscr{C}^{R}\right)$ and (ii) $A^{-1 / 4} C_{0}^{\infty}\left(\mathscr{C}^{R}\right)$ each are dense in $L_{\mathbb{R}}^{2}\left(\mathscr{C}^{R}, d x d^{2} \xi\right)$. (i) follows by Propositions 4.3 and A4.3-A4.6. For (ii) use $A: C_{0}^{\infty}\left(\mathscr{C}^{R}\right) \rightarrow C_{0}^{\infty}\left(\mathscr{C}^{R}\right)$ to argue $A^{-1 / 4} C_{0}^{\infty}\left(\mathscr{C}^{R}\right) C A^{-1 / 4} A C_{0}^{\infty}\left(\mathscr{C}^{R}\right)=A^{3 / 4} C_{0}^{\infty}\left(\mathscr{C}^{R}\right)$, which is dense by Propositions 4.3 and A4.3-A4.6.

\section{Discussion}

\subsection{Further Notes on the Relation to Other Work}

Our construction (Sect. 4) of $\left(k_{F}, h_{F}, \exp \left(-i h_{F} t\right)\right)$ corresponds to the original nonrigorous discussion (for the case $d=2, m>0$ ) of Fulling [2] (see also [31]). Fulling also gives an explicit spectral representation for $h_{F}$.

Theorem 2.1 was discovered at a heuristic level shortly after Hawking's "black hole evaporation" announcement (see [3, 4, 6-8] and also the review of Isham [32]). Theorem 2.1 can actually be extracted, at least formally, from the original work of Fulling [2] which gives an explicit form for the Bogolubov transformation $k_{0} \circ k_{F}^{-1}$ in terms of the spectral representation for $h_{F}$. However, in order to settle the domain questions which arise in making the argument rigorous, it appears that one in any case requires a good portion of the results obtained here. The heuristic analyticity argument given by Unruh et al. $[7,8,3,6]$ presumably has something to do with the analyticity arguments needed to prove Theorem 1.4 (see [17]) and the pre-Bisognano-Wichmann theorem (Sect. A1). However, our method for Theorem 2.1 is new. It is not the same as that in $[7,8,3,6]$ even at a formal level. An attempt to make the Unruh et al. discussion rigorous might proceed as follows: Show that $\left(\widetilde{R}_{F}^{2 \pi}, \widetilde{h}_{F}, e^{-i 2 T}\right)\left(\widetilde{k}_{F}^{2 \pi}, \widetilde{h}_{F}\right.$ as in Sect. 2) is a ground one-particle structure over $(D, \sigma, \mathscr{T}(T))$, where $\mathscr{T}(T)$ is ordinary time-evolution, and then invoke Theorem 1.1. The problem with making sense of such an argument is that $\widetilde{k}_{F}^{2 \pi}$ is a priori only defined on $\widetilde{D}$, whereas $\mathscr{T}(T)$ has to be defined on all of $D$. (It certainly does not map $\widetilde{D} \rightarrow \widetilde{D}$ !) Our present strategy (in terms of KMS rather than ground one-particle structures) works because it is always possible to restrict (in our case $k_{0}$ from $D$ to $\widetilde{D}$ ) but not necessarily to extend (i.e. $\widetilde{k}_{F}^{2 \pi}$ from $\widetilde{D}$ to $D$ )!

For other relevant early literature, note the reference in [8] to [33] which appears to be a non-rigorous forerunner of some of the "KMS-state aspects" of the problem discussed here (see discussion in [18]). I thank C. J. Isham for a discussion on this point [34].

10 To obtain a one-particle structure over the equivalent $\left(D^{R}, \sigma, \mathscr{V}(t)\right)$ of Sects. 2 and 3 , one, of course, simply replaces $k$ here by $k$ where $k$ consists of $k$ composed with the map $\Phi \mapsto \overleftarrow{\Phi}$ described above 


\subsection{More About the Significance of this Work}

The main results of this paper are the construction given for $\omega_{H}$ (strong supporting evidence for the reasonableness of which is given by Theorem 2.1) and Theorem 3.2. It should be noted that nothing in this article depends on the value of the Hawking temperature and Theorem 3.2 is, of course, equally valid is we replace $\omega_{H}$ by $\tilde{\omega}_{B}^{\beta}$ for any $0<\beta<\infty$. (For discussions of why $\beta=8 \pi M$ is a preferred value, see in addition to the already quoted literature, Dimock and Kay's scattering-theory results [16] and Haag, Narnhofer, and Stein's discussion in terms of their "principle of local definiteness" [35].)

Just as $\omega_{H}$ is analogous (by the Rindler-Fulling-Unruh analogy discussed in Sect. 0) to $\omega_{0}$, so Theorem 3.2 is analogous to familiar results for quantum fields in Minkowski space. In proving the results in Theorem 3.2, we have isolated a set of properties which are common to both (Minkowski and Schwarzschild) situations and which suffice for both (Minkowski and Schwarzschild) sets of results. In checking that these properties are actually possessed by the KleinGordon equation on the Schwarzschild spacetime, we have developed several tools which we hope will also be useful - in providing a constructive check for the case of linear fields - for investigations into other aspects of the Hawking effect.

\section{Appendices}

\section{A1. One-Particle Equivalents}

\section{to the Reeh-Schlieder and Bisognano-Wichmann Theorems}

We state and outline proofs for the one-particle versions of the Reeh-Schlieder and Bisognano-Wichmann theorems which are quoted in Sect. 2. Basic references for this section are the original papers [36] (see also [37]) and [12] for the full theorems. We shall also briefly explain the link between our one-particle versions and the full theorems.

Pre-Reeh-Schlieder Theorem. Let $\left(k_{0}, h_{0}, \exp \left(-i h_{0} T\right)\right)$ be the usual ground oneparticle structure over $(D, \sigma, \mathscr{T}(T))$ (see Sect. 2) and let $D_{\mathcal{O}} C D$ consists of Cauchy data with support in an arbitrary open set $\mathcal{O}$ of $\mathbb{R}^{3}$. Then $k_{0} D_{\mathcal{O}}+i k_{0} D_{\mathcal{O}}$ is dense in $h_{0}$.

Proof. Assume given some $\chi \in h_{0}$ such that $\langle\lambda \mid \chi\rangle=0 \quad \forall \lambda \in k_{0} D_{0}$. Then it remains to show $\chi=0$. Realizing $h_{0}$ as $L_{\mathbb{C}}^{2}\left(\mathbb{R}^{3}\right)$, then any $\lambda \in k_{0} D_{\mathbb{0}}$ may be written as $2^{-1 / 2}\left(\mu^{1 / 2} f+i \mu^{-1 / 2} p\right)$ with $(f, p) \in D_{0}$. For any such $\lambda$ [i.e. for any such $\left.(f, p)\right]$, define the (continuous, bounded ${ }^{11}$ ) function $F$ of $T, \mathbf{X}$ by

$$
F(T, \mathbf{X})=\left\langle\mu^{1 / 2} f+i \mu^{-1 / 2} p \mid e^{(i \mu T+\mathbf{X} \cdot \nabla)} \chi\right\rangle \quad(\mathbf{X}=(X, \xi)) .
$$

One easily sees that

$$
F(T, \mathbf{X})=\left\langle\mu^{1 / 2} f_{T, \mathbf{X}}+i \mu^{-1 / 2} p_{T, \mathbf{x}} \mid \chi\right\rangle,
$$

11 To see this, use the $K$-space realization of $h_{0}$ to express $F(T, \mathbf{X})$ as $\int d^{3} \mathbf{K} \overline{\tilde{\lambda}}(\mathbf{K}) \tilde{\chi}(\mathbf{K}) e^{i \mu(\mathbf{K}) T-i \mathbf{K} \cdot \mathbf{X}}$ $(\tilde{\lambda}, \tilde{\chi}$ the Fourier transforms of $\lambda, \chi)$ and apply the Riemann-Lebesgue lemma 
where $\left(f_{T, \mathbf{x}}, p_{T, \mathbf{x}}\right)$ are Cauchy data for the solution translated by $(T, \mathbf{X})$. By finite propagation speed and our assumption on $\chi$, it follows that $F(T, \mathbf{X})=0$ for all $(T, \mathbf{X})$ in some neighbourhood of zero. On the other hand, one easily sees ${ }^{12}$ that $F$ is the boundary value in the sense of distributions of a holomorphic function in $\mathbb{R}^{4}+i V_{+}$, where $V_{+}$is the usual forward cone $\left\{(T, \mathbf{X}) \in \mathbb{R}^{4}: T>|\mathbf{X}|\right\}$. One concludes by the edge-of-the-wedge theorem (Theorem 2.17 of [37]) that $F(T, \mathbf{X})$ vanishes for all $T, \mathbf{X}$. Specializing to translations, we may conclude that $\left\langle\mu^{1 / 2} f_{0, \mathbf{x}}+i \mu^{-1 / 2} p_{0, \mathbf{x}} \mid \chi\right\rangle=0$ for arbitrary translates of any $(f, p) \in D_{\mathcal{O}}$, and hence (e.g. by using a partition of unity) for all $(f, p) \in D$. Now use the fact that $k_{0} D$ is dense to conclude $\chi=0$.

Comments. (1) In the present paper, we use this theorem in the special case $\mathcal{O}=\mathbb{R}^{+} \times \mathbb{R}^{2}$. (2) This theorem is so named since an immediate consequence is that in the representation $W(\Phi) \mapsto W^{\mathscr{F}}\left(k_{0} \Phi\right)$ on $\mathscr{F}\left(h_{0}\right)$ of the Weyl algebra over $D$, the vacuum is cyclic for the algebra generated by $\left\{W(\Phi): \Phi \in D_{0}\right\}$. For this, use the fact about $W^{\mathscr{F}}(\cdot)\left([38]\right.$ as quoted in the theorem in Sect. A4 of [17]) that $\Omega^{\mathscr{F}}$ is cyclic for $\left\{W^{\mathscr{F}}(\chi): \chi \in M\right\}-M$ a real-linear subspace of $h$ - if and only if $M+i M$ is dense in $h$.

Pre-Bisognano-Wichmann Theorem. Let $\left(k_{0}, h_{0}, \exp \left(-i h_{0} T\right)\right)$ be the usual ground one-particle structure over $(D, \sigma, \mathscr{T}(T))$ and let $\exp \left(-i \kappa_{0} t\right)$ be the usual one-particle representation of the Lorentz boosts $\mathscr{V}(t)$ (see Sect. 2). Let $j_{0}$ be the complex conjugation on $h_{0}$ defined by $j_{0} \chi=C_{0} \check{\chi}$ (see Sect. 2). Then

$$
k_{0} D^{R}+i k_{0} D^{R} C \mathscr{D}\left(\exp \left(-\pi \kappa_{0}\right)\right), \quad k_{0} D^{L}+i k_{0} D^{L} C \mathscr{D}\left(\exp \left(\pi \kappa_{0}\right)\right)
$$

and

$$
\exp \left(-\pi \kappa_{0}\right) \chi^{R}=-j_{0} \chi^{R} \quad \forall \chi \in k_{0} D^{R}, \quad \exp \left(\pi \kappa_{0}\right) \chi^{L}=-j_{0} \chi^{L} \quad \forall \chi^{L} \in k_{0} D^{L} .
$$

Proof. It is convenient to realize $h_{0}$ as $L_{\mathbb{C}}^{2}\left(\mathbb{R}_{3}\right)$ (i.e. functions in momentum space). Thus

$$
k_{0}(f, p)(\mathbf{K})=2^{-1 / 2}\left(\mu^{1 / 2}(\mathbf{K}) \tilde{f}(\mathbf{K})+i \mu^{-1 / 2}(\mathbf{K}) \tilde{p}(\mathbf{K})\right) .
$$

Writing this as $\tilde{\chi}(\mathbf{K})$, it is not difficult to see that our Lorentz boosts act as

$$
\left(\exp \left(-i \kappa_{0} t\right) \tilde{\chi}\right)(\mathbf{K})=\mu^{-1 / 2}(\mathbf{K})\left(\mu^{1 / 2} \tilde{\chi}\right)(\Lambda(t) \mathbf{K}) \quad\left(\mu(\mathbf{K})=\left(\mathbf{K}^{2}+m^{2}\right)^{1 / 2}\right),
$$

where, in the expression $\Lambda(t) \mathbf{K}$, one takes for granted the obvious correspondence $\mathbf{K} \leftrightarrow(\mu(\mathbf{K}), \mathbf{K})$ between three-vectors and on-mass-shell four vectors. Putting in the details of our $\Lambda(t)$, we have

$$
\begin{aligned}
& \left\{\exp \left(-i t \kappa_{0}\right) k_{0}(f, p)\right\}\left(K_{1}, K_{2}, K_{3}\right) \\
& \quad=\left(2 \mu^{-1 / 2}\right)\left(K_{1}, K_{2}, K_{3}\right)\left\{(\mu \tilde{f}+i \tilde{p})\left(\mu(\mathbf{K}) \sinh t+K_{1} \cosh t, K_{2}, K_{3}\right)\right\} .
\end{aligned}
$$

We take the case of $D^{R}$, ( $D^{L}$ is similar). For any $(f, p) \in D^{R}, f\left(\cdot, K_{2}, K_{3}\right)$, $p\left(\cdot, K_{2}, K_{3}\right.$ ) are (for fixed $K_{2}, K_{3}$ ) boundary values of functions holomorphic in the lower half $K_{1}$ plane. Also (exclude for the moment the case $K_{2}=K_{3}=m=0$ )

12 To see this, use the previous footnote to express $F$-considered as a tempered distribution - as the 4-dimensional Fourier transform of $\delta\left(K_{0}-\mu(\mathbf{K})\right) \tilde{\lambda}(\mathbf{K}) \tilde{\chi}(\mathbf{K})$. Since this has support in the forward cone in momentum space, we may apply Theorem 2.9 of [37] to express its Fourier transform as the boundary value in the stated sense of the Laplace transform. (Note that this part of the proof goes through equally well in the case $m=0, d=2$; see Sect. A2) 
$\mu\left(\cdot, K_{2}, K_{3}\right)$ can be locally analytically continued away from the real axis [and has branch points at $\left.\pm i\left(K_{2}{ }^{2}+K_{3}{ }^{2}+m^{2}\right)^{1 / 2}\right]$. Relying on technical considerations similar to those in [12], we may now calculate $\left(\exp \left(-\pi \kappa_{0}\right) \tilde{\chi}\right)\left(K_{1}, K_{2}, K_{3}\right)$ as the result of analytically continuing along the image of the path $t: 0 \rightarrow-i \pi$ in the expression (*) above. The result is easily seen to be

$$
2^{-1 / 2}\left\{-\left(\mu^{1 / 2} \tilde{f}\right)\left(-K_{1}, K_{2}, K_{3}\right)+i\left(\mu^{-1 / 2} \tilde{p}\right)\left(-K_{1}, K_{2}, K_{3}\right)\right\} .
$$

The first minus sign occurs because this analytic continuation (whether we start at $K_{1}>0$ or $\left.K_{1}<0\right)$ clearly sends $\mu\left(K_{1}, K_{2}, K_{3}\right)$ to $-\mu\left(-K_{1}, K_{2}, K_{3}\right)$. [In the case $K_{2}=K_{3}=m=0$ or if $d=2$ and $m=0-$ see Sect. A2 $-\mu\left(K_{1}, K_{2}, K_{3}\right)$ reduces to $\left|K_{1}\right|$. We may still calculate in the same way (say excluding now the point $K_{1}=0$ ) calculating separately for $K_{1}>0$ (where $\left|K_{1}\right|$ analytically continues to $K_{1}$ ) and $K_{1}<0$ (where $\left|K_{1}\right|$ analytically continues to $-K_{1}$ ). Continuation along our path clearly sends $\left|K_{1}\right|$ to $-\left|K_{1}\right|$ in each case, so we still end up with the same expression.] One easily sees that this expression is just $-j_{0} k_{0}(f, p)\left(K_{1}, K_{2}, K_{3}\right)$. The full statement of the theorem easily follows by complex linearity of $\exp \left(-\pi \kappa_{0}\right)$.

Comment. Under second quantization, the expression $\exp \left(-\pi \kappa_{0}\right) \chi=-\mathrm{j}_{0} \chi$ yields the expression $\Delta_{0}^{1 / 2} W^{\mathscr{F}}\left(k_{0} \Phi\right)=J_{0} W^{\mathscr{F}}\left(k_{0} \Phi\right)^{*}$ for $\Phi \in D^{R}$, where $\Delta_{0}^{i t / 2 \pi}$ $=\Gamma\left(\exp \left(-i \kappa_{0} t\right)\right)$, and $J_{0}=\Gamma\left(j_{0}\right)$. [Use $W^{\mathscr{F}}(\chi)^{*}=W^{\mathscr{F}}(-\chi)$.] This is what we call the Bisognano-Wichmann theorem in Sect. 2.

\section{A2. Notes on the Minkowski Cases $m=0, d \neq 4$ (Especially $m=0, d=2$ )}

We discuss to what extent the results of Sect. 2 (and the relevant parts of Sects. 4 and A1) generalize to $d$-dimensional Minkowski space and to the case $m=0$. First, it is easy to see that, provided $m>0$, everything generalizes straightforwardly to all $d \geqq 2$. If $m=0, d \geqq 3$, then the construction of the ordinary Minkowski vacuum goes through unmodified as do the results in Sect. A1. However, it is not clear whether the construction of a regular ground one-particle structure of Sect. 4 (and in consequence Theorem 2.1) will go through without modification because of the failure of the estimate (4.7). We leave this question open ${ }^{13}$. We shall, however, now explain how to treat the "most severe" case $m=0, d=2$, where modification is certainly necessary.

This case, the wave equation

$$
\left(\frac{\partial^{2}}{\partial T^{2}}-\frac{\partial^{2}}{\partial X^{2}}\right) \phi=0
$$

on $\mathbb{R}^{214}$ plays an important role (as the "inner free dynamics") in the scattering theory of (3.1) (see [16] and also Sect. A3 here) and in [16] we will require the modified version of Theorem 2.1 given below for this (2-dimensional!) equation in

13 Postscript: Fulling and Ruijsenaars [45] have since taken up this question and claim that no modification is required in the cases $m=0, d \geqq 3$

14 Actually, on $\mathbb{R}^{2} \times \mathbb{S}^{2}$ - but with $\xi \in \mathbb{S}^{2}$ not entering in the equation. The only change this makes is that we should replace $\mathscr{C} \approx \mathbb{R}$ by $\mathscr{C} \approx \mathbb{R} \times \mathbb{S}^{2}, d X$ by $d X d^{2} \xi$, and $d x$ by $d x d^{2} \xi$ in what follows (where $d^{2} \xi$ is the usual measure on $\mathbb{S}^{2}$ ) 
calculating the behaviour on the horizon of the Hartle-Hawking and Unruh states on (4-dimensional!) Schwarzschild spacetime. As is well known [39], there are difficulties with defining the Minkowski vacuum in the 2-dimensional massless case. (Essentially, one can only define vacuum expectation values of products of derivatives of $\hat{\phi}$.) From our point of view (see Sect. 2), the problem is that $k_{0}$ cannot be defined on all of $D$ because $C_{0}^{\infty}(\mathscr{C})\left(\approx\right.$ in $X$-coordinates $\left.C_{0}^{\infty}(\mathbb{R})\right) \not \mathscr{D}\left(\mu^{-1 / 2}\right)$. (Use: in momentum space $\mu=|K|$.) To overcome this, we borrow an idea from Streater and Wilde $[40]$ and replace $D$ by the domain $\hat{D}=\left\{(f, p) \in C_{0}^{\infty}(\mathscr{C}) \times \hat{C}_{0}^{\infty}(\mathscr{C})\right\}$, where by $\hat{C}_{0}^{\infty}(\mathscr{C})$ we mean $\left\{p \in C_{0}^{\infty}(\mathscr{C}): \int_{-\infty}^{\infty} p(X) d X=0\right\}$.

Similarly, we replace $D^{R}$ by $D^{R}=D^{R} \cap \hat{D}, D^{L}$ by $\hat{D}^{L}=D^{L} \cap \hat{D}$ and $\tilde{D}$ by $\hat{D}^{\sim}=\tilde{D} \cap \hat{D}$. One now has that $\hat{C}_{0}^{\infty}(\mathscr{C}) \subset \mathscr{D}\left(\mu^{-1 / 2}\right)$. [In fact, one easily sees that

$$
\hat{C}_{0}^{\infty}(\mathscr{C}) \subset \mathscr{D}\left(\mu^{-1}\right)
$$

so that $k_{0}$ now makes sense on $\hat{D}$ and in fact, $\left(k_{0}, L_{\mathbb{C}}^{2}(\mathbb{R}, d X), \exp \left(-i h_{0} T\right)\right.$ ) (with $h_{0}=\mu$ - which is manifestly strictly positive) is a ground one-particle structure for $(\hat{D}, \sigma, \mathscr{T}(T))$.$] We define algebras \hat{\mathfrak{A}}, \hat{\mathfrak{A}}^{L}, \hat{\mathfrak{U}}^{R}, \hat{\mathfrak{U}}^{\sim}$ generated by $\{W(\Phi)$ : $\Phi \in \hat{D}, \hat{D}^{L}, \hat{D}^{R}, \hat{D}^{\sim}$, respectively $\}$ and define $\omega_{0}$ by $(2.8)$ on $\hat{\mathfrak{A}}$.

Proofs of the pre-Bisognano-Wichmann and pre-Reeh-Schlieder theorems (Sect. A1) still go through (when suitably modified by $d X d^{2} \xi \equiv d^{3} \mathbf{X} \rightarrow d X, D \rightarrow \hat{D}$ ). For pre-Bisognano-Wichmann, this was already mentioned in Sect. A1. For preReeh-Schlieder, the only new feature (cf. the penultimate sentence of the proof) reduces to showing that, given an arbitrary interval $\mathcal{O}$ of $\mathbb{R}$, an arbitrary function $p \in \hat{C}_{0}^{\infty}(\mathbb{R})$ can be written as a finite sum of translates of functions in $\hat{C}_{0}^{\infty}(\mathbb{R})$ which each have their support in $\mathcal{O}$. It is not difficult to see that this is true.

Finally, although estimate (4.7) no longer holds, it is easy to see that - replacing $D^{R}$ by $\hat{D}^{R}-$ a construction of a regular ground one-particle structure $\left(k_{F}, h_{F}, e^{-i h_{F} t}\right)$ along the lines of Sect. 4 still goes through. In fact, under the change of coordinates (4.1), (4.4) becomes $-\frac{\partial^{2}}{\partial x^{2}}\left(=\mu^{2}\right)$ while the map (Sect. 4) $\Phi \mapsto \overleftarrow{\Phi}$ defined by $(f, p) \mapsto\left(f, e^{x} p\right)$ maps $\hat{D}$ into the subspace of $\bar{D} \hat{D}^{<}=\left\{\widehat{\Phi} \in \widehat{D}: \int_{-\infty}^{\infty} \hat{p} d x=0\right\}$, so that $\left(\hat{D}^{<}, \delta, \overleftarrow{\mathscr{V}}(t)\right)$ is in fact just a copy ${ }^{15}$ [with $(t, x)$ coordinates on $\mathscr{R}$ replacing $(T, X)$ coordinates an $\mathscr{M}]$ of $(\hat{D}, \sigma, \mathscr{T}(T))$. The $\left(\grave{k}_{F}, h_{F}, \exp \left(-i h_{F} t\right)\right)$ of Sect. 4 also turns out (as it must) to be a copy (with the same replacements) of the $\left(k_{0}, h_{0}, \exp \left(-i h_{0} T\right)\right)$ already discussed above, and Proposition 4.4 and Theorem 4.5 remain true when modified by the replacements $d x d^{2} \xi \rightarrow d x$ and $\bar{D} \rightarrow \hat{D}^{<}$. In particular, $\hat{D}^{<} \subset \mathscr{D}\left(A^{1 / 2}\right) \oplus \mathscr{D}\left(A^{-1 / 2}\right)$ amounts to (A2.2).

Putting everything together as in Sect. 2, we can thus conclude

Theorem 2.1 $\left(d=2, m=0\right.$ Version). On $\hat{\mathfrak{A}}^{\sim}, \omega_{0}=\tilde{\omega}_{F}^{2 \pi}$.

\section{A3. A Proof that $\mathbf{s p}\left(\boldsymbol{h}_{\boldsymbol{B}}\right)=[0, \infty)$}

By Sect. 4, we can take $h_{B}=A_{B}^{1 / 2}$ on $h_{B}=L_{\mathbb{C}}^{2}\left(\mathscr{C}^{R} \approx \mathbb{R} \times \mathbb{S}^{2}, d x d^{2} \xi\right)$, where $A_{B}$ is as in (4.5). Since $A_{B}$ is clearly positive, it suffices to show $[0, \infty) \subset \operatorname{sp}\left(h_{B}\right)$. We offer a proof

15 The reason is that $\left(\frac{\partial^{2}}{\partial T^{2}}-\frac{\partial^{2}}{\partial X^{2}}\right) \phi=0$ becomes, in $(t, x)$ coordinates, $\left(\frac{\partial^{2}}{\partial t^{2}}-\frac{\partial^{2}}{\partial x^{2}}\right) \phi=0$ ! 
here (cf. Theorems VI.1 and VI.2 in [41]) which exhibits this as a consequence of the classical scattering theory developed by Dimock and Kay for (3.1) combined with the one-particle-structure machinery of Sect. 1.1, and the results of Sect. 4. (No doubt alternative proofs - in the form $[0, \infty) \subset \operatorname{sp}\left(A_{B}\right)$ - are possible by more Schrödinger-operator-style methods.)

Consider the multiplication operator $k$ on the Hilbert space $h^{+}=L_{\mathbb{C}}^{2}\left(\mathbb{R}^{+} \times \mathbb{S}^{2}, d k d^{2} \xi\right) . k$ trivially has $\operatorname{sp}(k)=[0, \infty)$. We shall prove our result by showing the existence of an invariant subspace ${ }^{16} h_{B}^{+}$of $h_{B}$ such that $h_{B}$ restricted to $h_{B}^{+}$is unitarily equivalent to $k$ on $h^{+}$. First, recall that $h_{B}$ figures in the oneparticle structure $\left(\hat{k}_{B}, h_{B}, \exp \left(-i h_{B} t\right)\right)$ over $\left(\mathscr{D}\left(A_{B}^{1 / 2}\right) \oplus \mathscr{D}\left(A_{B}^{-1 / 2}\right), \stackrel{\sigma}{,}, \exp \left(-\mathbf{h}_{B} t\right)\right)$ (see Proposition 4.4). We may view $k$ as figuring in a one-particle structure in the following parallel way: Let $\partial$ be the usual skew-adjoint operator $\partial / \partial x$ on $L_{\mathbb{R}}^{2}\left(\mathbb{R} \times \mathbb{S}^{2}, d x d^{2} \xi\right)$. Defining $\forall f_{1}, f_{2} \in \mathscr{D}(\partial)$,

$$
\sigma\left(f_{1}, f_{2}\right)=\left\langle f_{1} \mid \partial f_{2}\right\rangle-\left\langle f_{2} \mid \partial f_{1}\right\rangle
$$

one easily sees that $\left(\mathscr{D}(\partial), \sigma, e^{-t \partial}\right)$ is a classical linear dynamical system. Moreover, defining

$$
k: \mathscr{D}(\partial) \mapsto h^{+}=L_{\mathbb{C}}^{2}\left(\mathbb{R}^{+} \times \mathbb{S}^{2}, d k d^{2} \xi\right)
$$

by $(k f)(k, \xi)=2^{1 / 2} k^{1 / 2} \tilde{f}(k, \xi) \quad\left(\right.$ where $\tilde{f}(k, \xi)=(2 \pi)^{-1 / 2} \int_{-\infty}^{\infty} f(x, \xi) e^{-i k x} d x$ and restriction to $\mathbb{R}^{+}$is understood $)$, one checks that $\left(k, h^{+}, e^{-i k t}\right)$ is a ground oneparticle structure over $\left(\mathscr{D}(\partial), \sigma, e^{-t \hat{\partial}}\right)$.

Dimock and Kay have proved ${ }^{17}$ the existence of an operator

$$
\Omega: \mathscr{D}(\partial) \rightarrow \mathscr{D}\left(A_{B}^{1 / 2}\right) \oplus \mathscr{D}\left(A_{B}^{-1 / 2}\right)
$$

satisfying

$$
\delta\left(\Omega f_{1}, \Omega f_{2}\right)=\sigma\left(f_{1}, f_{2}\right) \quad \forall f_{1}, f_{2} \in \mathscr{D}(\partial)
$$

and intertwining $\mathbf{h}_{B}$ with $\partial$ in the sense that

$$
\Omega \exp \left(-\mathbf{h}_{B} t\right)=\exp (-t \partial) \Omega .
$$

Using these properties (and Proposition 4.4 as quoted above) one may check that

$$
\left(\hat{k}_{B} \circ \Omega, h_{B}^{+}, \exp \left(-i h_{B} t\right) \uparrow_{h_{B}^{+}}\right),
$$

$16 h_{B}^{+}$will turn out (see footnote 17 below) on reading [16] to be interpretable as the part of $h_{B}$ which "falls through the past horizon"

17 To make the link with [16], one must note that the map $f \mapsto(f,-\partial f)$ defines an equivalence between $\left(\mathscr{D}(\partial), \sigma, e^{-t \partial}\right.$ ) and the "right-going part" (in the sense of [16]) of the $d=2, m=0$ Minkowski version (in the sense of footnote 14) of Sect. A2 of $\left(\mathscr{D}\left(A^{1 / 2}\right) \oplus \mathscr{D}\left(A^{-1 / 2}\right), \tilde{\sigma}, \exp (-\mathbf{h} t)\right)$. Also, under this same equivalence, $\left(k, h^{+}, e^{-i k t}\right)$ is then equivalent to the "right-going part" [16] of (the same $d=2, m=0$, Sect. A2 version of ) the $\left(\widehat{k}_{F}, h_{F}, \exp \left(-i h_{F} t\right)\right)$ of Proposition 4.4

Finally, the $\Omega$ here is then equivalent to the "right-going inner wave operator $\Omega_{1}^{-}$" of $[16]$. The quoted properties of $\Omega$ then correspond to results stated for $\Omega_{1}^{-}$in [16]. (Our full proof of these statements is omitted from [16] but appears in the case $m=0$ in [42]. The case $m>0$ is similar.) Note finally that we could, of course, have worked equally well with $\Omega_{1}^{+}$ 
where

$$
h_{B}^{+}=\overline{\operatorname{ran} \kappa_{B} \circ \Omega} \subset h_{B}
$$

is an alternative one-particle structure over $\left(\mathscr{D}(\partial), \sigma, e^{-t \partial}\right)\left[h_{B}^{+}\right.$is clearly invariant under $\left.\exp \left(-i h_{B} t\right)\right]$. To see that it is complex-linear, apply Theorem 1.2 to the corresponding projection.

We may thus apply Theorem 1.1 to conclude the existence of a unitary $U: h^{+} \rightarrow h_{B}^{+}$satisfying

$$
h_{B} \uparrow_{h_{B}^{+}}=U k U^{-1} \text {. }
$$

This fulfills our promise.

\section{A4. Some Results on Self-Adjoint and Positive-Self-Adjoint Operators Required for Sect. 4}

In order to make the logic of the proofs in Sect. 4 clear, we explicitly state the facts about self-adjointness, and about positive-self-adjoint operators to which we shall need to appeal there. We adopt the convention below that $S$ stands for a skewsymmetric operator on some (separable, real) Hilbert space, while $T$ stands for a self-adjoint, and $P$ and $Q$ for positive-self-adjoint operators on some (separable, real or complex) Hilbert space. Where no proofs or references are given, the results are elementary consequences of results in e.g. [43].

Proposition A4.1. Suppose there exists a strongly continuous orthogonal group $O(t): \mathscr{D}(S) \rightarrow \mathscr{D}(S)$ with strong derivative $\frac{d O}{d t}(t) \uparrow_{t=0}=-S$ on $\mathscr{D}(S)$. Then $S$ is essentially skew-adjoint. (Proof by e.g. [43, Theorem VIII.10] and complexification.)

Proposition A4.2. Suppose that, in addition to the hypotheses of A4.1, $S: \mathscr{D}(S) \rightarrow \mathscr{D}(S)$. Then, all odd (even) powers of $S$ are skew-(self-)adjoint on $\mathscr{D}(S)$.

Proof. See Chernoff [29].

Proposition A4.3. If $T$ has dense range, and $\Delta$ is a core for $T$, then $\operatorname{ran}\left(T \uparrow_{\Delta}\right)$ is also dense.

Proposition A4.4. $P$ is strictly positive (i.e. $P$ has no zero eigenvalues) $\Leftrightarrow \operatorname{ran} P$ is dense.

Proposition A4.5. $P$ strictly positive $\Leftrightarrow P^{\alpha}$ strictly positive, $\alpha=\frac{1}{4}, \frac{1}{2}, \frac{3}{4}$, etc.

Proposition A4.6. Any core $\Delta$ for $P$ is a core for $P^{\alpha}, \alpha=\frac{1}{4}, \frac{1}{2}, \frac{3}{4}$, etc.

Proposition A4.7. ${ }^{18}$ A sufficient condition for $P$ strictly positive is the existence of $a$ core $\Delta$ for $P$ and a strictly positive $Q$ such that $\Delta \subset \mathscr{D}(Q)$ and $\langle x \mid P x\rangle \geqq\langle x \mid Q x\rangle$ $\forall x \in \Delta$.

For the proof of A4.7, use A4.5, A4.6, and the following

18 The use of such a proposition in Sect. 4 arose from a suggestion of J. Dimock 
Lemma A4.8. Let $\Delta \subset \mathscr{D}(P) \cap \mathscr{D}(Q)$ be a core for $P$, then $\|P x\| \geqq\|Q x\|$ $\forall x \in \Delta \Rightarrow \mathscr{D}(P) \subset \mathscr{D}(Q)$ and $\|P x\| \geqq\|Q x\| \forall x \in \mathscr{D}(P)$.

Finally, we have

Proposition A4.9. In the situation of Proposition $A 4.7, \mathscr{D}\left(Q^{-1 / 2}\right) \subset \mathscr{D}\left(P^{-1 / 2}\right)$.

For the proof of A4.9, use A4.6, A4.8, A4.4, and the following two lemmas.

Lemma A4.10. $\|P x\| \geqq\|Q x\| \quad \forall x \in \mathscr{D}(P) \Rightarrow \mathscr{D}\left(P^{1 / 2}\right) \subset \mathscr{D}\left(Q^{1 / 2}\right)$ and $\left\|P^{1 / 2} x\right\|$ $\geqq\left\|Q^{1 / 2} x\right\| \forall x \in \mathscr{D}\left(P^{1 / 2}\right)$ (see the "monotonicity of the square root" in [44]).

Lemma A4.11. Let $B, C$ be densely defined operators such that $\operatorname{ran} C \subset \mathscr{D}(B)$ and $B C$ is bounded on $\mathscr{D}(C)$. Then $\operatorname{ran} B^{*} C \mathscr{D}\left(C^{*}\right)$ and $C^{*} B^{*}$ is bounded on $\mathscr{D}\left(B^{*}\right)$.

Acknowledgements. This work grew out of a project begun during a visit to the Istituto Matematico "G. Castelnuovo", Università di Roma, Italy in autumn-winter 1982-1983. I thank S. Doplicher for his kind invitation and the CNR-Gnafa for financial support. It was continued during the tenure (March-August 1983) of a McCormick fellowship at the Enrico Fermi Institute, University of Chicago. Here the work was supported by the McCormick Foundation and by the NSF (under grant no. PHY 80-26043). Support was also provided by the Schweizerischer Nationalfonds in Zürich.

I thank J. Dimock, S. Doplicher, S. A. Fulling, C. J. Isham, R. Longo, E. Müller, and G. Scharf for useful discussions and correspondence. Thanks also go to J. Dimock for permission to quote from our not yet published joint work on scattering theory on the Schwarzschild metric.

\section{References}

1. Hawking, S.W.: Commun. Math. Phys. 43, 199-220 (1975)

2. Fulling, S.A.: Nonuniqueness of canonical field quantization in Riemannian spacetime. Phys. Rev. D 7, 2850-2862 (1973)

3. Fulling, S.A.: Alternative vacuum states in static spacetimes with horizons. J. Phys. A10, 917-951 (1977)

4. Davies, P.C.W.: Scalar particle production in Schwarzschild and Rindler metrics. J. Phys. A 8, 609-616 (1975)

5. Hartle, J.B., Hawking, S.W.: Path-integral derivation of black-hole radiance. Phys. Rev. D 13, 2188-2203 (1976)

6. Hawking, S.W.: Breakdown of predictability in gravitational collapse. Phys. Rev. D 14, 2460-2473 (1976)

7. Unruh, W.G.: Notes on block-hole evaporation. Phys. Rev. D 14, 870-892 (1976)

8. Israel, W.: Thermo-field dynamics of black holes. Phys. Lett. 57 A, 107-110 (1976)

9. Boulware, D.G.: Quantum field theory in Schwarzschild and Rindler spaces. Phys. Rev. D 11, 1404-1423 (1975)

10. Birrell, N.D., Davies, P.C.W.: Quantum fields in curved space. Cambridge: Cambridge University Press 1982

11. Sewell, G.L.: Relativity of temperature and the Hawking effect. Phys. Lett. 79 A, 23-24 (1980); Quantum fields on manifolds: PCT and gravitationally induced thermal states. Ann. Phys. (NY) 141, 201-224 (1982)

12. Bisognano, J.J., Wichmann, E.H.: On the duality condition for a Hermitian scalar field. J. Math. Phys. 16, 985-1007 (1975); On the duality condition for quantum fields. 17, 303-321 (1976)

13. Kay, B.S.: Commun. Math. Phys. 71, 29-46 (1980)

14. Dimock, J.: Commun. Math. Phys. 77, 219 (1980) 
15. Kay, B.S.: Springer Lecture Notes in Mathematics 905, 272-295 (1982)

16. Dimock, J., Kay, B.S.: Classical and quantum scattering theory for linear scalar fields on the Schwarzschild metric (to appear)

17. Kay, B.S.: A uniqueness result for quasi-free KMS states. Helv. Phys. Acta (to appear)

18. Kay, B.S.: Purification of KMS states. Helv. Phys. Acta (to appear)

19. Slawny, J.: Commun. Math. Phys. 24, 151-170 (1972)

20. Kay, B.S.: A uniqueness result in the Segal-Weinless approach to linear Bose fields. J. Math. Phys. 20, 1712-1713 (1979)

21. Weinless, M.: J. Funct. Anal. 4, 350 (1969)

22. Connes, A.: Ann. Sci. Ec. Norm. Sup. $4^{\mathrm{e}}$ série 6, 133 (1973)

23. Hawking, S., Ellis, G.: The large scale structure of spacetime. Cambridge: Cambridge University Press 1973

24. Geroch, R.: Domain of dependence. J. Math. Phys. 11, 437-449 (1970)

25. Leray, J.: Hyperbolic partial differential equations. Princeton Lecture Notes. Princeton University 1952 (mimeographed)

26. Choquet-Bruhat, Y.: Hyperbolic partial differential equations on a manifold. In: Battelle rencontres. de Witt, B.S., Wheeler, J.A., eds. New York: Benjamin 1967

27. Kay, B.S.: Commun. Math. Phys. 62, 55-70 (1978)

28. Kay, B.S.: Springer Lecture Notes in Physics 116, 241-243 (1980)

29. Chernoff, P.R.: J. Funct. Anal. 12, 401-414 (1973)

30. Reed, M., Simon, B.: Methods of modern mathematical physics. Vol. III: Scattering theory. New York, London: Academic Press 1979

31. Candelas, P., Raine, D.: Quantum field theory on incomplete manifolds. J. Math. Phys. 17, 2101-2112 (1976)

32. Isham, C.J.: Ann. N.Y. Acad. Sci. 302, 114-157 (1977)

33. Takahashi, Y., Umezawa, H.: Collect. Phenom. 2, 55-80 (1975)

34. Isham, C.J.: Private communication (1976)

35. Haag, R., Narnhofer, H., Stein, U.: Commun. Math. Phys. 94, 219-238 (1984)

36. Reeh, H., Schlieder, S.: Bemerkungen zur Unitäräquivalenz von Lorentz invarianten Feldern. Nuovo Cimento 22, 1051-1068 (1961)

37. Streater, R.F., Wightman, A.S.: PCT spin, statistics and all that. New York: Benjamin 1964

38. Leyland, P., Roberts, J., Testard, D.: Duality for quantum free fields. Marseille preprint (1978)

39. Wightman, A.S.: Introduction to some aspects of the relativistic dynamics of quantum fields. Cargèse lectures in theoretical physics: high energy electromagnetic interactions and field theory, Lévy, M., ed. (1964). New York: Gordon and Breach 1967

40. Streater, R.F., Wilde, I.: Fermion states of a boson field. Nucl. Phys. B 24, 561-575 (1970)

41. Dimock, J., Kay, B.S.: Classical wave operators and asymptotic quantum field operators on curved spacetimes. Ann. Inst. Henri Poincaré 37, 93-114 (1982)

42. Dimock, J.: Scattering for the wave-equation on the Schwarzschild metric. General Relativity and Gravitation (to appear)

43. Reed, M., Simon, B.: Methods of modern mathematical physics, Vol. I: Functional analysis. New York, London: Academic Press 1972

44. Kato, T.: Perturbation theory for linear operators. Berlin, Heidelberg, New York: Springer 1966

45. Fulling, S., Ruijsenaars, S.: Texas $A$ and $M$ mathematics preprint (unpublished)

Communicated by S. W. Hawking

Received November 12, 1984; in revised form January 12, 1985 
